\title{
Detrital zircon U-Pb geochronology and geochemistry of the Riachuelos and Palma Sola beach sediments, Veracruz State, Gulf of Mexico: a new insight on palaeoenvironment
}

\author{
John S. Armstrong-Altrin(i)
}

\begin{abstract}
Zircons are abundant in the beach sediments. In this study, surface microtexture, mineralogy, bulk sediment geochemistry, trace element composition and U-Pb isotopic geochronology of detrital zircons collected from the Riachuelos and Palma Sola beach areas, southwestern Gulf of Mexico were performed to infer the sediment provenance and palaeoenvironment. The zircon microtexture was categorized as mechanically- and/or chemicallyinduced features. The weathering index values for the Riachuelos $(\sim 72-77)$ and Palma Sola $(\sim 71-74)$ beach sediments indicated moderate weathering of both of the two source areas. The major and trace element data of bulk sediments suggested passive margin settings for the two areas. The trace elemental ratios and chondritenormalized rare earth element (REE) patterns of bulk sediments revealed that the sediments were likely sourced by felsic and intermediate igneous rocks. And the zircon Th/U ratios (mostly more than 0.2 ) and zircon REE patterns (with negative Eu and positive Ce anomalies) suggested a magmatic origin for both of the beach sediments from these two areas. Two distinct zircon age peaks respectively belonging to the Paleozoic and the Cenozoic were identified both in the Riachuelos and Palma Sola beach sediments. Zircon geochronology comparison research between the Riachuelos-Palma Sola beach sediments and potential source areas in SW Gulf of Mexico revealed that the source terrane supplied the Paleozoic zircons of this study was identified as the Mesa Central Province $(\mathrm{MCP})$, and the Cenozoic zircons were transported from the nearby Eastern Alkaline Province (EAP). Moreover, although the Precambrian zircons were very few in the studied sediments, their geochronology and geochemistry results still could infer that they were contributed by the source terranes of Grenvillian igneous suites in the Oaxaca and the Chiapas Massif Complexes.
\end{abstract}

Keywords: Detrital zircon, Beach sediment, U-Pb dating, Zircon grain morphology, Microtexture, Mineralogy, Geochemistry, Geochronology, Gulf of Mexico

Correspondence: armstrong@cmarl.unam.mx; john_arms@yahoo.com

Unidad de Procesos Oceánicos y Costeros, Instituto de Ciencias del Mar y

Limnología, Universidad Nacional Autónoma de México, Ciudad Universitaria,

04510 Ciudad de México, Mexico

\section{Springer Open}

(c) The Author(s). 2020 Open Access This article is licensed under a Creative Commons Attribution 4.0 International License, which permits use, sharing, adaptation, distribution and reproduction in any medium or format, as long as you give appropriate credit to the original author(s) and the source, provide a link to the Creative Commons licence, and indicate if changes were made. The images or other third party material in this article are included in the article's Creative Commons licence, unless indicated otherwise in a credit line to the material. If material is not included in the article's Creative Commons licence and your intended use is not permitted by statutory regulation or exceeds the permitted use, you will need to obtain permission directly from the copyright holder. To view a copy of this licence, visit http://creativecommons.org/licenses/by/4.0/. 


\section{Introduction}

Zircon is a common accessory mineral in clastic sediments, which retains $\mathrm{U}-\mathrm{Pb}$ isotopic signatures during erosion, recycling, and transport; differences in zircon age populations in sediments are linked to the nature and age of source terranes (Ji et al. 2019; Joy et al. 2019; Liu et al. 2019; Chaudhuri et al. 2020). Hence, detrital zircon $\mathrm{U}-\mathrm{Pb}$ age geochronology is reliable to infer provenance, as well as helpful to discriminate sediments derived from various source terranes and to understand their sediment-transport pathways (An et al. 2016;
Madhavaraju et al. 2018; Wang et al. 2018a). Although $\mathrm{U}-\mathrm{Pb}$ ages of detrital zircons have been used widely in different studies to investigate sediment provenance, few studies recommended that the combination of zircon age data and trace element data is more reliable to better understand the depositional history of a complex depositional basin (Wang et al. 2017; Li et al. 2019).

On the other hand, the importance of mineralogical and geochemical composition of clastic sediments to infer intensity of weathering, provenance, and tectonic setting of the source region is well established in the

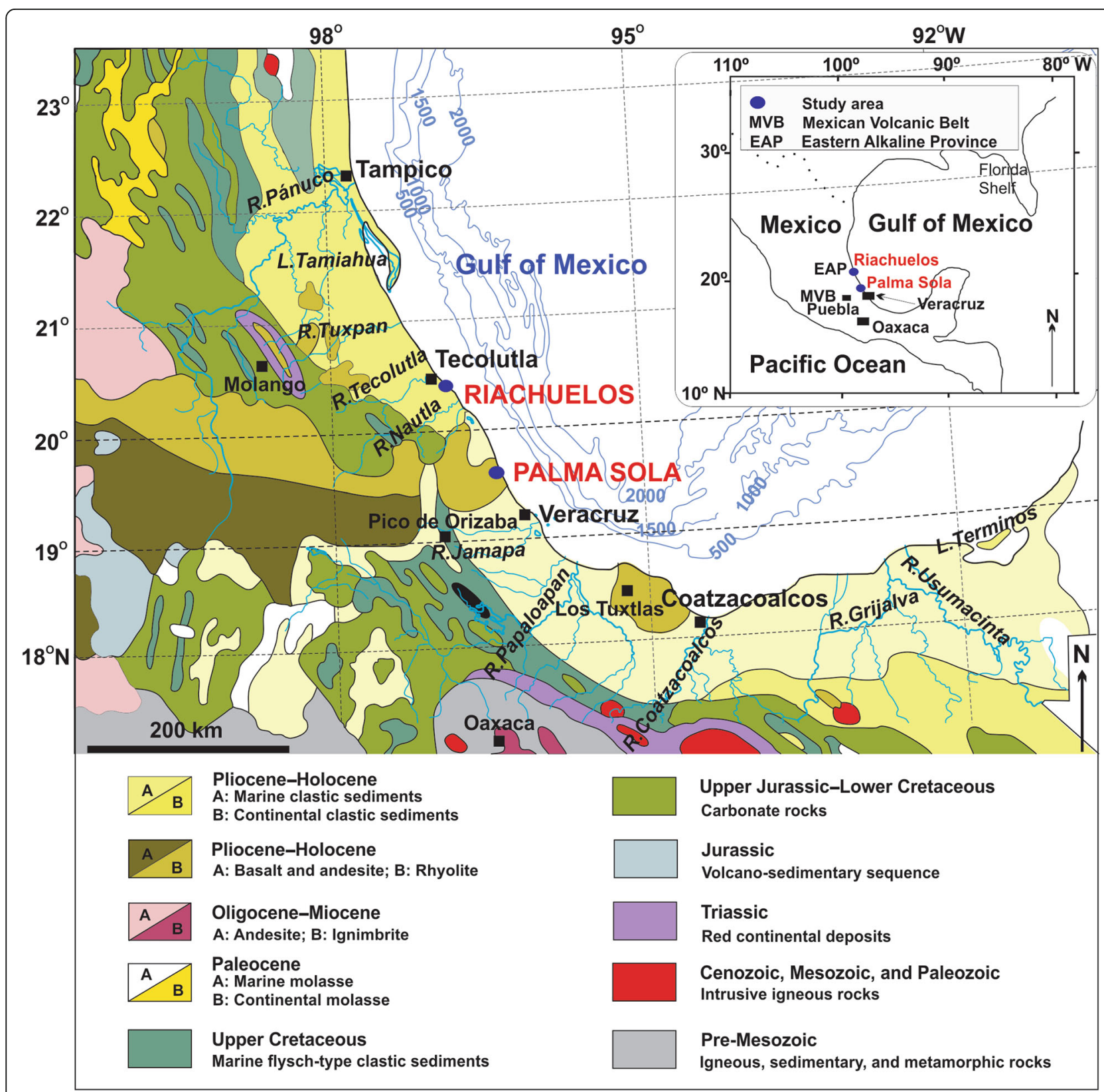

Fig. 1 Map showing the geology and geography of Riachuelos and Palma Sola beach areas, Gulf of Mexico (Source: Carta Geológica; Scale: 1:1,000,000; modified after Anaya-Gregorio et al. 2018). L. = Lagoon; R. = River 
literature (Basu et al. 2016; Carranza-Edwards et al. 2019; Casse et al. 2019; Karudu 2019; Men et al. 2019; Kettanah et al. 2020). Although clastic sediments are affected by factors such as weathering, diagenesis, and mineral fractionation during transport, their mineralogical-geochemical composition is mainly related to the parent rock types and the influence factors of the source (McLennan et al. 1993; Basu 2017; Ramos-Vázquez et al. 2017, 2018; Bansal et al. 2018; Ndjigui et al. 2019; Rivera-Gómez et al. 2020). Therefore, the geochemical composition of clastic sediments is also reliable to infer the tectonic setting of a sedimentary basin. The immobile trace elements and their elemental ratios differ widely between felsic and mafic rocks, hence are particularly helpful to discriminate the sediments derived from felsic (high in $\mathrm{Zr}, \mathrm{Nb}, \mathrm{Hf}, \mathrm{Y}$, and $\mathrm{Th}$ ) or mafic (high in $\mathrm{Cr}, \mathrm{V}, \mathrm{Ni}$, and $\mathrm{Sc}$ ) sources (Cullers and Podkovyrov 2002; Tawfik et al. 2017). Similarly, different source rocks can be identified by the value of Eu anomaly, for instance, sediments derived from mafic igneous rocks, especially basalt, exhibit positive $\mathrm{Eu}$ anomalies or no $\mathrm{Eu}$ anomaly (Basu 2017; Wang et al. 2018b; L wen et al. 2020). In addition, the mobile elements like $\mathrm{K}, \mathrm{Na}$, and $\mathrm{Ca}$ among major elements are useful to infer the weathering intensity and other palaeoenvironmental conditions of the source area (Barros dos Santos et al. 2019).

Textural characteristics and geochemical compositions of beach sediments along the Gulf of Mexico have been studied by some authors (Rosales-Hoz et al. 2008;
Tapia-Fernandez et al. 2017; Hernández-Hinojosa et al. 2018; Ramos-Vázquez et al. 2018). And many studies have been focussed on the zircon $\mathrm{U}-\mathrm{Pb}$ ages of volcanic rocks from various terranes in Mexico, like Chiapas, Oaxaca, Zacatecas, and Xolapa, with little consideration to the detrital zircons in beach sediments (Keppie et al. 2003; Weber et al. 2009, 2018; Talavera-Mendoza et al. 2013; Escalona-Alcázar et al. 2016; Cisneros de León et al. 2017; Peña-Alonso et al. 2017, 2018; Ramírez-Peña and Chávez-Cabello 2017). Recently, based on the geochemical composition of sandstone and the detrital zircon U-Pb geochronology, Wengler et al. (2019) interpreted the provenance of a sedimentary unit in the Mesa Central region, central Mexico. However, investigations based on the comprehensive research of mineralogy, detrital zircon $\mathrm{U}-\mathrm{Pb}$ geochronology, major and trace element geochemistry of bulk sediment in the Gulf of Mexico beach area to infer the sediment provenance, are meagre.

The degree of grain roundness and microtextures on quartz grain surfaces are considered as powerful tools to infer sediment provenance and have been applied in various studies to reconstruct palaeoenvironments (Margolis and Krinsley 1974; Mahaney 2002; Madhavaraju et al. 2009; Mahaney et al. 2012; Vos et al. 2014; Kalińška-Nartiša et al. 2018; Chmielowska and Woronko 2019). However, microtextures, especially on zircon grains, are not studied sufficiently, possibly because of the difficulties in separating zircon grains from rocks

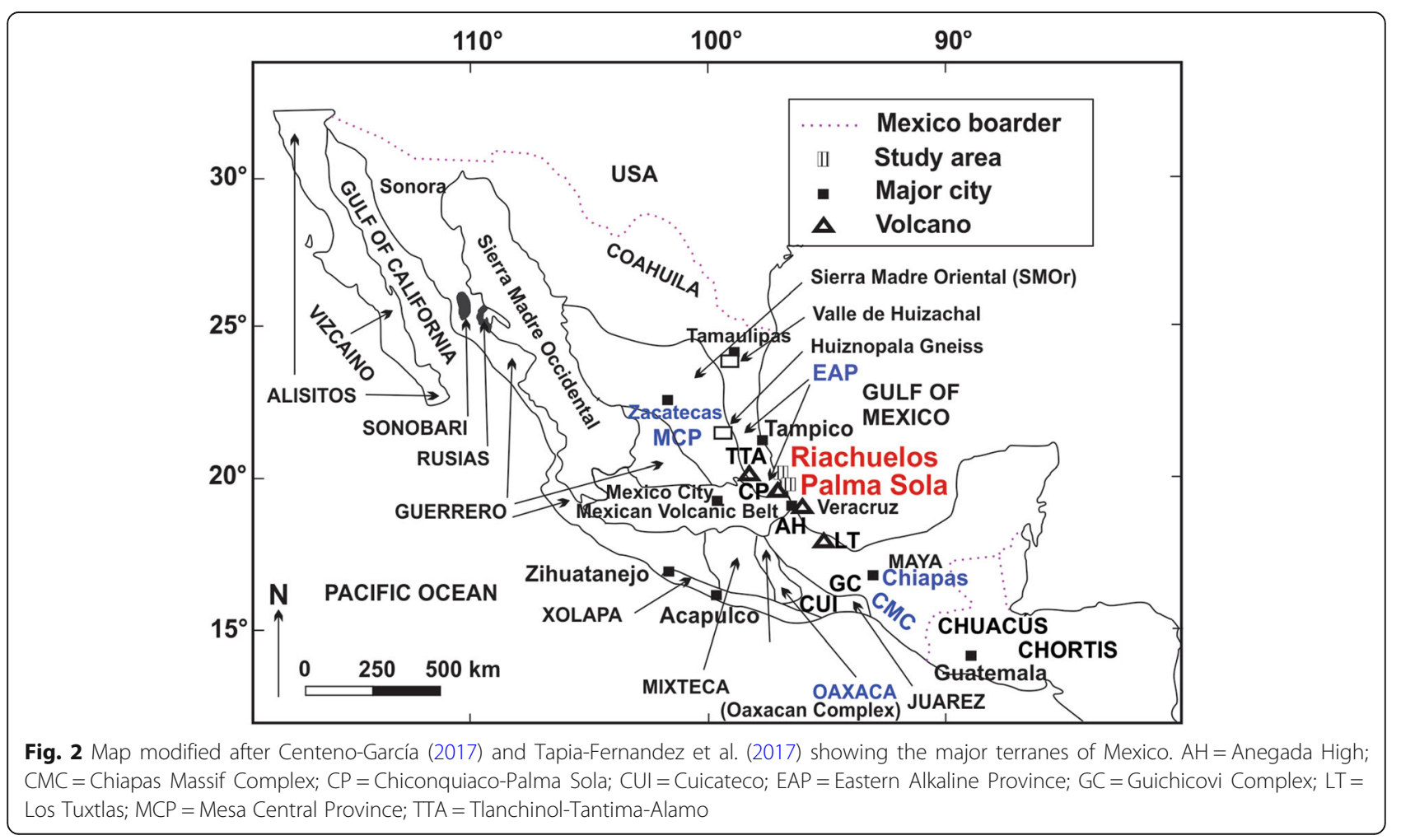


and sediments. To fulfil this gap, the microtextures of detrital zircons from the Riachuelos and Palma Sola beach sediments are surveyed, and the provenance information based on the types of surface features is briefly discussed.

Furthermore, in this study, the mineralogy and geochemistry of bulk sediments, $\mathrm{U}-\mathrm{Pb}$ and trace element data of detrital zircons collected from the Riachuelos and Palma Sola beach areas are analysed, to investigate the source areas supplying sediments to the beach areas and to infer the palaeoenvironments.

\section{Geological setting}

The Riachuelos and Palma Sola beach areas are located at the Veracruz State, SW Gulf of Mexico $\left(20^{\circ} 25^{\prime} 16.88^{\prime \prime}\right.$ $\mathrm{N}-96^{\circ} 57^{\prime} 28.00^{\prime \prime} \mathrm{W}$ and $19^{\circ} 46^{\prime} 24.73^{\prime \prime} \mathrm{N}-96^{\circ} 25^{\prime} 19.30^{\prime \prime} \mathrm{W}$, respectively; Fig. 1).

The geology of coastal regions of the Gulf of Mexico is shown in Fig. 1. The outcrops along the Gulf of Mexico coast are mainly composed of the PrecambrianPaleozoic metamorphic rocks comprising schist and gneiss, the Mesozoic-Cenozoic clastic and calcareous sedimentary rocks, the Cenozoic mafic and intermediate volcanic rocks, and the Quaternary volcanic rocks and clastic sediments (Keppie et al. 2003; Kasper-Zubillaga et al. 2019).

The Gulf of Mexico littoral zone belongs to the wavedominated transgressive marginal sea coast with narrow coastal plain (Inman and Nordstrom 1971; Davis 1988; Boyd et al. 1992; Carranza-Edwards 2011). The Riachuelos coast is wider than the Palma Sola coast. The easternmost side of the Trans-Mexican Volcanic Belt (TMVB) ends at the Veracruz State area, which formed a cliff at northern Palma Sola beach (Verma et al. 2016), and conduced longshore surface currents of northward flow direction in summer with an average velocity of about $4.5 \mathrm{~cm} / \mathrm{s}$. Higher wind velocities were recorded during summer, varying from $3.0 \mathrm{~m} / \mathrm{s}$ to $5.4 \mathrm{~m} / \mathrm{s}$ (YañezArancibia and Day Jr. 1982). However, during winter, longshore surface currents flow towards south with an average velocity of $6 \mathrm{~cm} / \mathrm{s}$. Monreal-Gómez and Salas de León (1990) documented that Gulf of Mexico water circulation and hydrodynamic conditions are controlled by loop currents and anticyclonic rings. The climate for Gulf of Mexico is considered as sub-humid to humid conditions (Tamayo 1991).

The major rivers feeding sediment to the western and southern areas of the Gulf of Mexico are Pánuco,
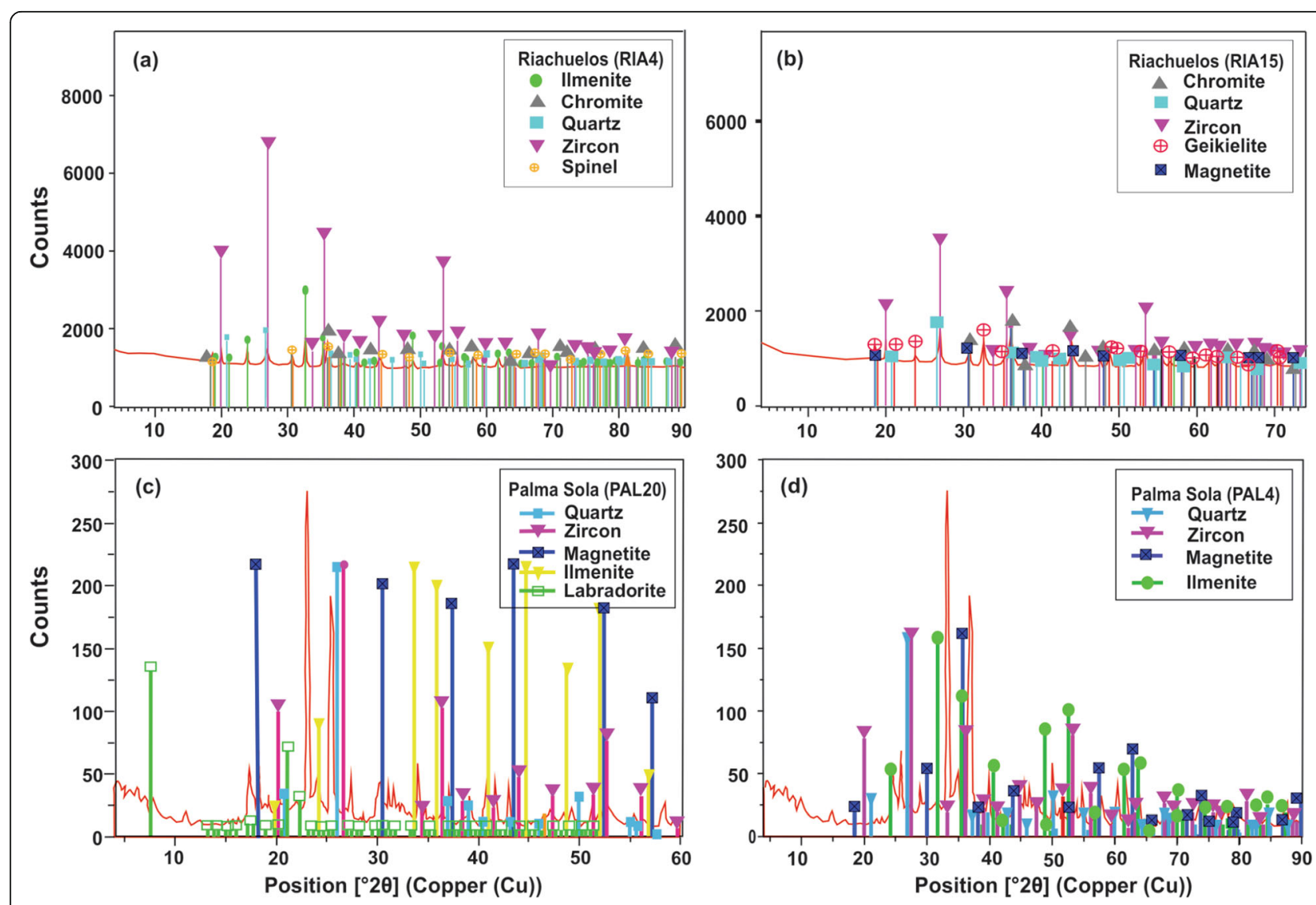

Fig. 3 X-Ray diffraction analysis result patterns for the Riachuelos $(\mathbf{a}, \mathbf{b})$ and Palma Sola $(\mathbf{c}, \mathbf{d})$ beach sediments 
Tecolutla, Jamapa, Papaloapan, Coatzacoalcos, Grijalva and Usumacinta (Fig. 1). River Pánuco is $510 \mathrm{~km}$ long with a drainage area of $\sim 98,227 \mathrm{~km}^{2}$, which drains into the Gulf of Mexico at Tampico State (Fig. 1). The drainage area of River Pánuco covers parts of Trans-Mexican Volcanic Belt (TMVB) and the Sierra Madre Oriental (SMOr) and its sediment lithology consists of Upper Jurassic-Lower Cretaceous carbonate rocks, Upper Cretaceous marine flysch-type clastic sediments and Pliocene-Holocene marine clastic sediments. River Tecolutla is one of the major rivers in Mexico, starts from the Xolapa uplift that is the easternmost extension of Mexican Volcanic Belt (MVB; Figs. 1 and 2), and, flows through and covers a large volcanic terrane (Self 1975) of an Neogene to Quaternary geological province extending through Central Mexico for about $1000 \mathrm{~km}$ (Verma 2009, 2015). River Jamapa flows for about 368 $\mathrm{km}$ from the Pico de Orizaba $\left(19^{\circ} 02^{\prime} \mathrm{N}\right.$ and $97^{\circ} 16^{\prime} \mathrm{W}$; volcano Citlalépetl) eastward emptying into the Gulf of Mexico at Boca del Rio, Veracruz State. The sediment lithology of River Jamapa draining area is dominated by the Upper Cretaceous marine flysch, and the PlioceneHolocene volcanics and marine clastic sediments (Fig. 1). River Papaloapan joins in the Gulf of Mexico at Alvarado, Veracruz State, with an annual water discharge of $39,175,000 \mathrm{~m}^{3}$ (Tamayo 1991). The drainage area of River Papaloapan is the second largest hydrological basin $\left(17^{\circ}-19^{\circ} \mathrm{N}\right.$ and $\left.95^{\circ}-97^{\circ} \mathrm{W}\right)$ in Mexico, which partly covers the Cuicateco terrane $\left(39,189 \mathrm{~km}^{2}\right.$; Fig. 2$)$. River Coatzacoalcos originates in the Oaxaca State area with a mean annual discharge of $32,732 \mathrm{hm}^{3}$ (Tamayo 1991), and generates a drainage basin of between $17^{\circ} 46^{\prime}-18^{\circ} 10^{\prime} \mathrm{N}$ and $92^{\circ} 25^{\prime}-94^{\circ} 31^{\prime} \mathrm{W}$. The sediment lithology of the River Coatzacoalcos draining area consists mainly of intrusive igneous rocks and metasedimentary rocks of the Oaxacan Complex. River Grijalva flows for about $640 \mathrm{~km}$ from the Chiapas State area of southeastern Mexico. River Usumacinta, originated from the northwestern region of neighbouring Guatemala, is considered as the longest river of Mexico with $\sim 1100 \mathrm{~km}$ length, and ranks second among the freshwater rivers flowing into the Gulf of Mexico (Muñoz-Salinas et al.
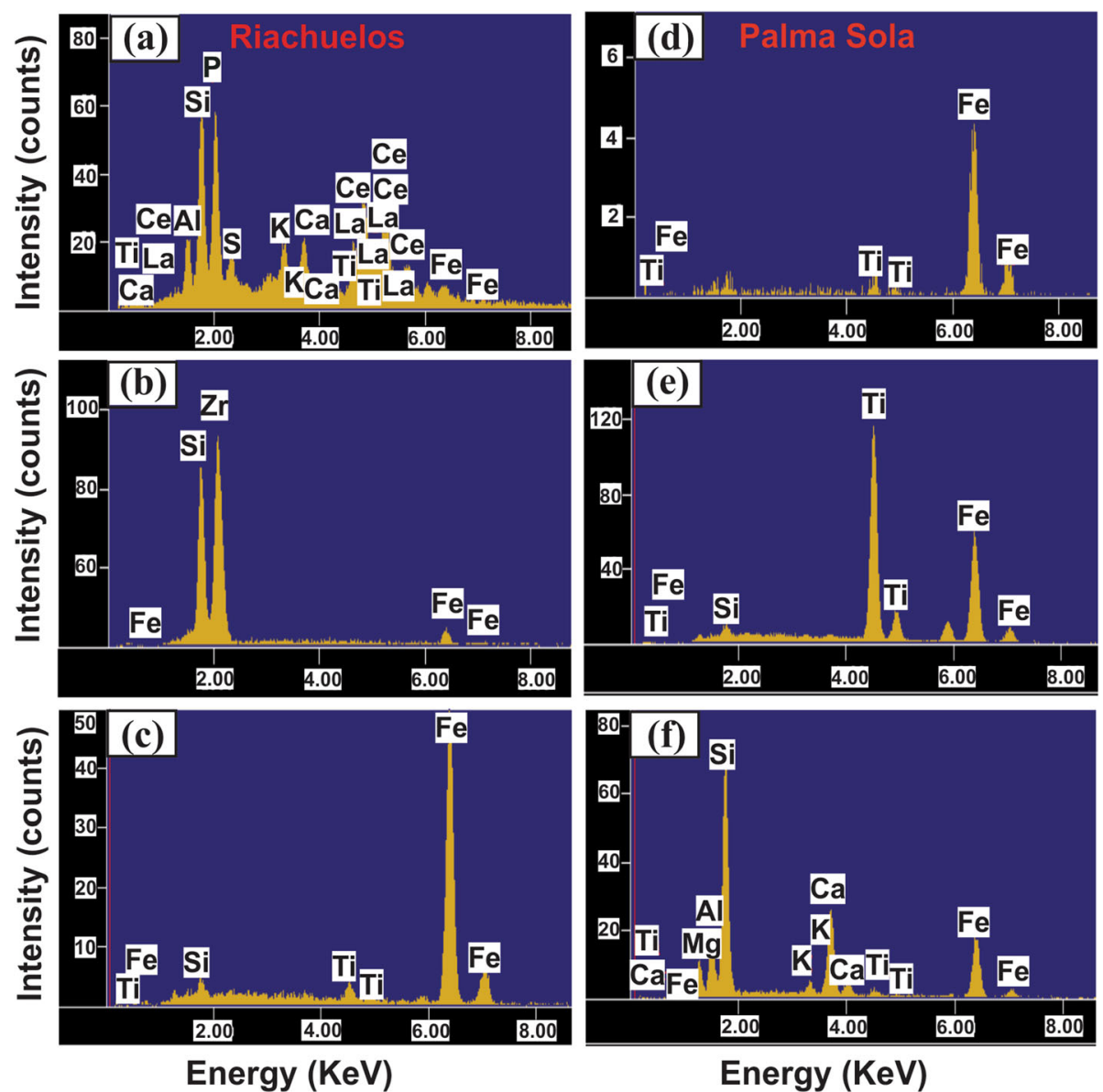

Fig. 4 Energy dispersive spectrums of a Apatite in Sample RIA1; b Zircon in Sample RIA14; c Magnetite in Sample RIA15 of the Riachuelos beach sediments; and d Titanomagnetite in Sample PAL5; e Ilmenite in Sample PAL13; f Quartz in Sample PAL20 of the Palma Sola beach sediments 
2017). The Grijalva and Usumacinta Rivers join upstream in about $50 \mathrm{~km}$ before they enter the Gulf of Mexico together (Muñoz-Salinas et al. 2017).

\section{Material and methods}

In total, 35 bulk sediment samples $(\sim 2 \mathrm{~kg}$ each $)$ were collected from the Riachuelos (number of samples $n=$ $15)$ and Palma Sola $(n=20)$ beach areas, where the waves reach the coast during high tide.

The mineralogy of 10 sediment samples in different size fractions (fine- and medium-grained) was identified using the Shimadzu XRD-6000 diffractometer at the
Institute of Geology, Universidad Nacional Autónoma de México (UNAM). X-Ray diffraction (XRD) was operated with an accelerating voltage of $40 \mathrm{kV}$ and a filament current of $30 \mathrm{~mA}$, using $\mathrm{CuK \alpha}$ radiation and a graphite monochromator.

Scanning Electron Microscopy (SEM) was used to infer the morphological features and shapes of the zircon grains. Fifteen zircon grains were randomly handpicked from each sediment sample under a stereomicroscope, and then 150 zircon grains were selected for microtexture analysis. Grains coated with gold and palladium were mounted on $3 \mathrm{~mm}$-thick carbon discs and

\section{Riachuelos}

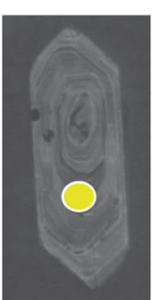

27 $282 \pm 7 \mathrm{Ma}$

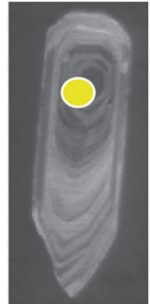

32

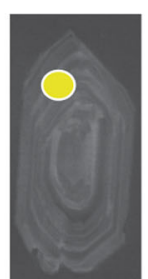

40 $3.3 \pm 0.5 \mathrm{Ma}$

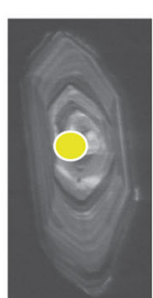

43 $4 \pm 1 \mathrm{Ma}$

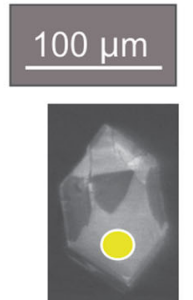

48 $282 \pm 7 \mathrm{Ma}$

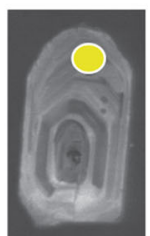

49 $13 \pm 1 \mathrm{Ma}$

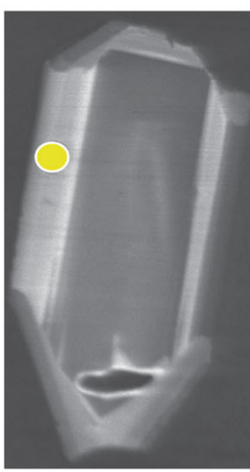

50

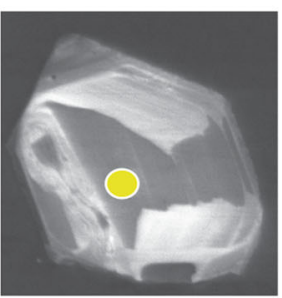

51

$280 \pm 7 \mathrm{Ma}$

$12.7 \pm 0.8 \mathrm{Ma}$

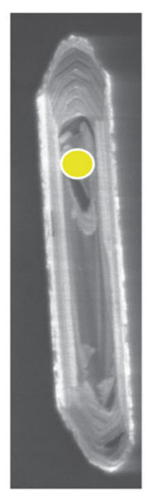

55

$13 \pm 1 \mathrm{Ma}$

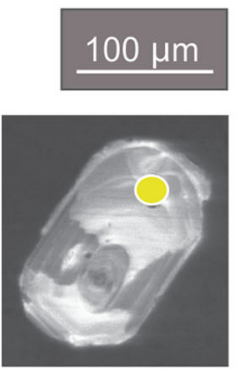

52

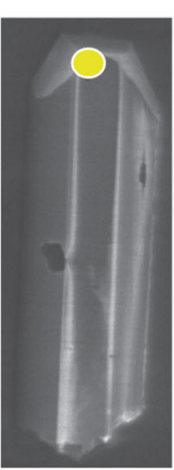

54

$12.3 \pm 1.3 \mathrm{Ma} \quad 5.0 \pm 2.6 \mathrm{Ma}$

\section{Palma Sola}

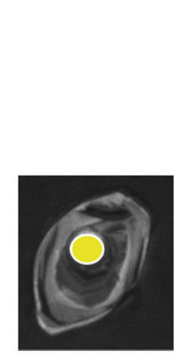

13

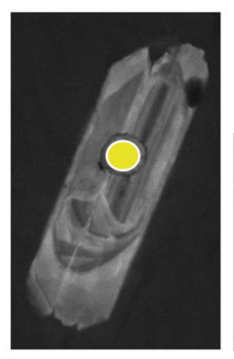

15

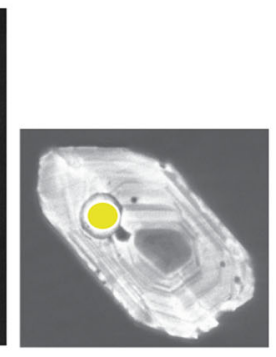

24

\section{$1496 \pm 30 \mathrm{Ma} \quad 292 \pm 8 \mathrm{Ma} \quad 271.8 \pm 6.4 \mathrm{Ma}$}

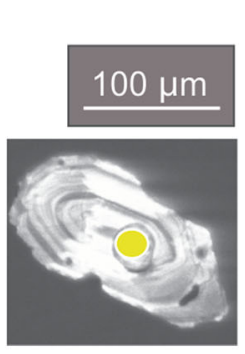

25

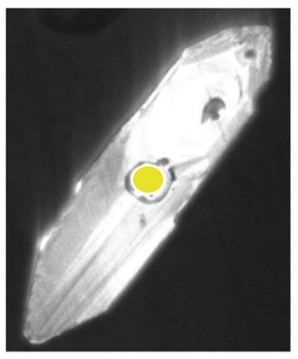

29

Fig. 5 Cathodoluminescence images of representative detrital zircon grains collected from the Riachuelos and Palma Sola beach sediments. The yellow circles on the images represent the LA-ICP-MS analytical sites (33 $\mu \mathrm{m})$ 
examined by JEOL-JSM-6360LV SEM with general magnifications between $\times 180$ and $\times 5500$ at Instituto de Ciencias del Mar y Limnología, UNAM. The quantitative compositional data of randomly-selected zircon grains (7 grains in each sample) were determined by JEOL-JXA8900R SEM equipped with Energy Dispersive X-Ray Spectrometry (SEM-EDS) at UNAM.

Major element concentration of total 35 bulk sediment samples collected from the Riachuelos and Palma Sola beach areas were analysed by Rigaku RIX-3000 X-Ray Fluorescence at the Institute of Geology, UNAM. Accuracy of major element analysis was monitored by the standard JGB1 (GSJ). The precision of major element data was better than $5 \%$. Loss on ignition was obtained by weighing after $1 \mathrm{~h}$ combustion at $1000{ }^{\circ} \mathrm{C}$. Trace element concentration of 30 bulk sediment samples were determined by a VG Elemental PQII plus ICP-MS and the operation procedure was similar as details in Jarvis (1988). The United States Geological Survey Standard BCR-2 (Basalt, Columbia River) was used for trace element data calibration, and the analytical precision was less than $5 \%$ in general.

Two bulk sediment samples, one from Riachuelos beach area (RIA4) and another from Palma Sola beach area (PAL20) were selected for detrital zircon U-Pb dating. In total, 263 detrital zircon grains (146 grains from the Riachuelos and 117 grains from the Palma Sola) were handpicked from bulk sediments, mounted in epoxy resins and polished. Cathodoluminescence images were taken using an ELM 3R luminoscope to reveal zircon internal texture. All 263 zircon grains were analysed for obtaining information of trace element $(\mathrm{Nb}, \mathrm{Hf}, \mathrm{Th}, \mathrm{U}, \mathrm{Pb})$ and REE geochemistry, and $\mathrm{U}-\mathrm{Pb}$ isotope geochronology.

Zircon $\mathrm{U}-\mathrm{Pb}$ dating and trace element concentration analyses were simultaneously conducted using a LAICP-MS coupled with Thermo Xii Series quadrupole mass spectrometry, followed by the methodology described by Solari et al. (2018). ANIST 610 glass standard was used to recalculate the trace element concentration, by normalizing them with ${ }^{29} \mathrm{Si}$. $\mathrm{U}$ and $\mathrm{Th}$ concentrations

Table 1 Microtextures of mechanical and chemical features identified on the zircon grain surfaces in the Riachuelos and Palma Sola beach sediments

\begin{tabular}{|c|c|c|c|}
\hline \multirow[t]{2}{*}{ Microtexture } & \multicolumn{2}{|l|}{ Zircon grain } & \multirow{2}{*}{ Palaeoenvironment $^{\mathrm{a}}$} \\
\hline & Riachuelos & Palma Sola & \\
\hline \multicolumn{4}{|l|}{ Mechanically-induced feature } \\
\hline Abraded edge (abe) & $x$ & $X X X$ & Aeolian, saltation, collision \\
\hline Dual striated zircon (dsz) & $x$ & $X X$ & Saltation, collision, short transport \\
\hline $\begin{array}{l}\text { Euhedral zircon with one side broken edge } \\
\text { (bez) }\end{array}$ & $X X$ & $x$ & Aeolian, saltation, collision, short transport, storm record \\
\hline Crescentic gouge (crg) & $x$ & & Near shore, wave action \\
\hline Arc-shaped step (as) and Linear step (Is) & $x$ & $X X$ & High-energy collision, aeolian, littoral zone, glacial zone \\
\hline Bulbous edge (ble) & $X X$ & & Aeolian, saltation, fluvial, dune \\
\hline Reworked conchoidal fracture (rcf) & $x$ & $X X$ & High-energy collision, aeolian, littoral zone, nearshore subaqueous \\
\hline Collision fracture (cf) & & $X X X$ & High-energy collision, aeolian, littoral zone \\
\hline Meandering ridge $(\mathrm{mr})$ & & $x$ & Aeolian, littoral dune, subaqueous \\
\hline V-shaped percussion crack (vs) & & $x$ & High-energy collision, gouging, littoral zone, deltaic, subaqueous, surf zone \\
\hline Straight groove (sgr) & $x$ & & Littoral zone, wave action, saltation \\
\hline \multicolumn{4}{|l|}{ Chemically-induced feature } \\
\hline Solution and precipitation feature (s/p) & $X X$ & $X X$ & Diagenetic environment, high in contaminated sea water (alkaline fluid) \\
\hline Circular solution pit (csp) & $X X$ & $x$ & Intertidal zone, diagenetic, percolation of sea water \\
\hline Grain cavities (gc) & & & Diagenetic, percolation of sea water \\
\hline Delamination (dl) & $x$ & $x$ & Collision/diagenetic \\
\hline Silica pellicle (sp) & $x$ & $x$ & Starting stage of in-situ diagenetic, nearshore \\
\hline $\begin{array}{l}\text { Adhered particle appears to be silica } \\
\text { globule (ads) }\end{array}$ & & $x$ & In-situ diagenetic, silica saturated, low-energy \\
\hline Silica flower (sf) and crystal overgrowth & & $x$ & $\begin{array}{l}\text { Advanced stage of diagenetic environment, silica oversaturated nearshore, } \\
\text { low-energy }\end{array}$ \\
\hline Adhered particle $(\mathrm{ad})^{\mathrm{b}}$ & $X X X$ & $X X X$ & Diagenetic, littoral, low-energy \\
\hline
\end{tabular}

XXX means Abundant; XX means Common; X means Present; ${ }^{a}$ stands for citations after Mahaney (2002), Madhavaraju et al. (2009), Mahaney et al. (2012), Armstrong-Altrin and Natalhy-Pineda (2014), Vos et al. (2014), Hossain et al. (2020), and Mohammad et al. (2020); ${ }^{\text {b }}$ Adhered particles are defined as mechanical/ chemical origin in some studies (e.g. Madhavaraju et al. 2009; Vos et al. 2014). Referring Figs. 6 and 7 for SEM images 
are calculated by employing an external standard zircon as mentioned in Paton et al. (2010). ${ }^{207} \mathrm{~Pb} /{ }^{206} \mathrm{~Pb}$ ratios, ages and errors are calculated according to Petrus and Kamber (2012). Individual analyses with >10\% uncertainty, $>20 \%$ discordance, or $>5 \%$ reverse discordance were not considered for interpretation. The $\mathrm{U}-\mathrm{Pb}$ concordia-age plots, the probability density distribution histograms were plotted using ISOPLOT software
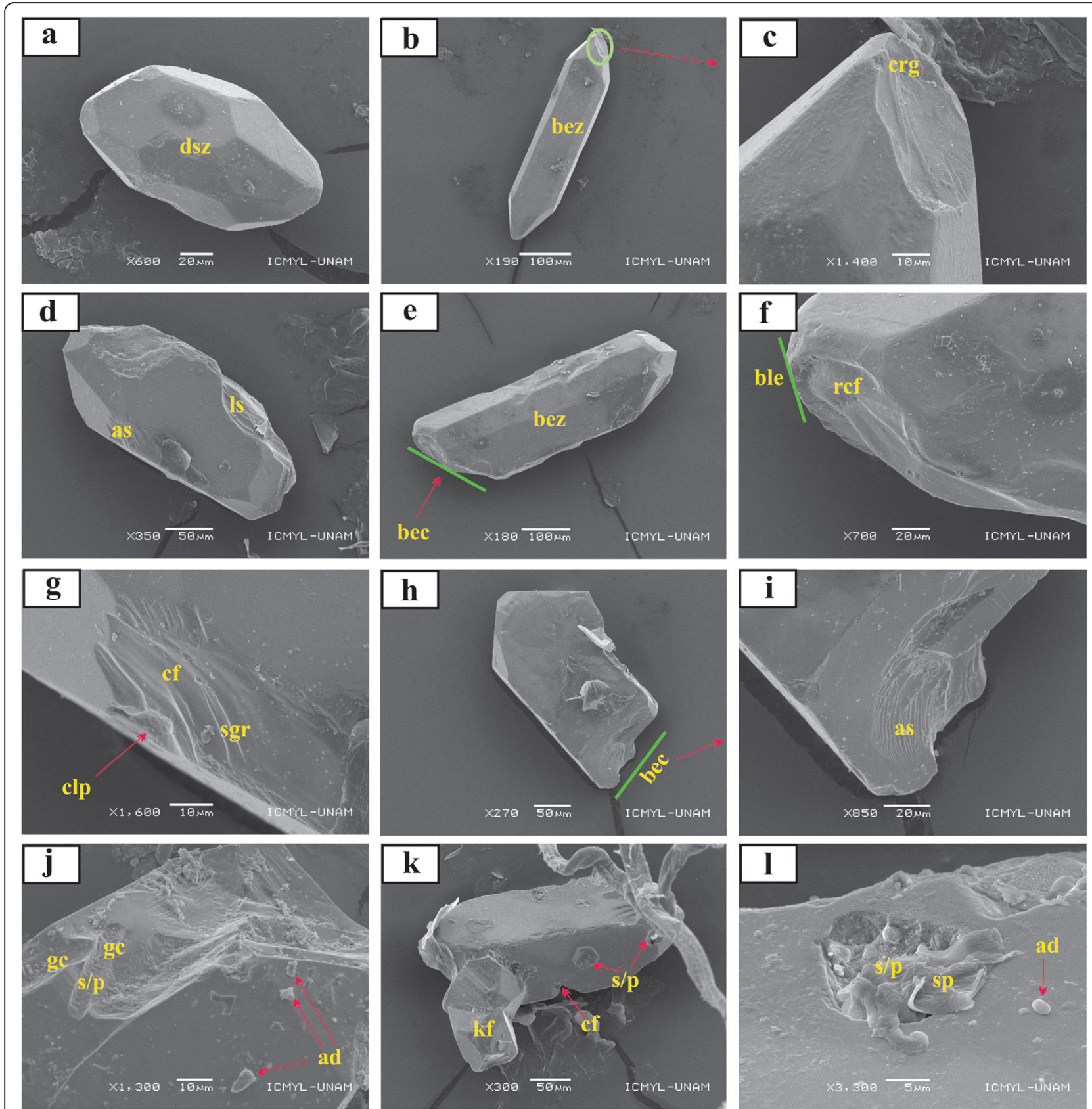

Fig. 6 Scanning Electron Microscopy (SEM) images showing surface microtextures of detrital zircons from the Riachuelos beach sediments. a Dual striated zircon (dsz) well abraded at both ends; $\mathbf{b}$ Euhedral zircon with one side broken edge (bez); $\mathbf{c}$ Enlarged view of the broken edge of grain b showing crescentic gouge (crg); d Grain showing arc-shaped steps (as) and linear steps (Is); e Euhedral zircon with one side broken edge (bez), probably due to collision (bec); $\mathbf{f}$ Zircon grain with bulbous edge (ble) and reworked conchoidal fracture ( $\mathrm{rcf}$ ); $\mathbf{g}$ Grain showing collision impact point (clp), collision fracture (cf) with straight groove (sgr); $\mathbf{h}$ Grain with broken edge due to collision (bec); $\mathbf{i}$ Enlarged view of broken edge of grain $\mathbf{h}$ showing arc-shaped steps (as); j Grain with cavities (gc), adhered particles (ad), solution and precipitation features (s/p); k K-feldspar (kf) adhering to a zircon grain, also showing collision fracture ( $(\mathrm{f})$, and solution and precipitation features (s/p); I Grain with silica pellicle (sp), adhered particle (ad), and solution and precipitation features $(\mathrm{s} / \mathrm{p})$ 
(Ludwig 2008). Further detailed analytical methods followed to measure zircon $\mathrm{U}-\mathrm{Pb}$ isotopes by LA-ICPMS were the same as descriptions in Solari et al. (2018).

Comparison research between the detrital zircon U$\mathrm{Pb}$ dating ages tested in this study with the previously published age data (Cantagrel and Robin 1979; LópezInfanzón 1991; Nelson and González-Caver 1992; Ferrari et al. 2005; Weber et al. 2009, 2018; Solari et al. 2011; Ortega-Obregón et al. 2014; Escalona-Alcázar et al. 2016; Cisneros de León et al. 2017; Wengler et al. 2019)
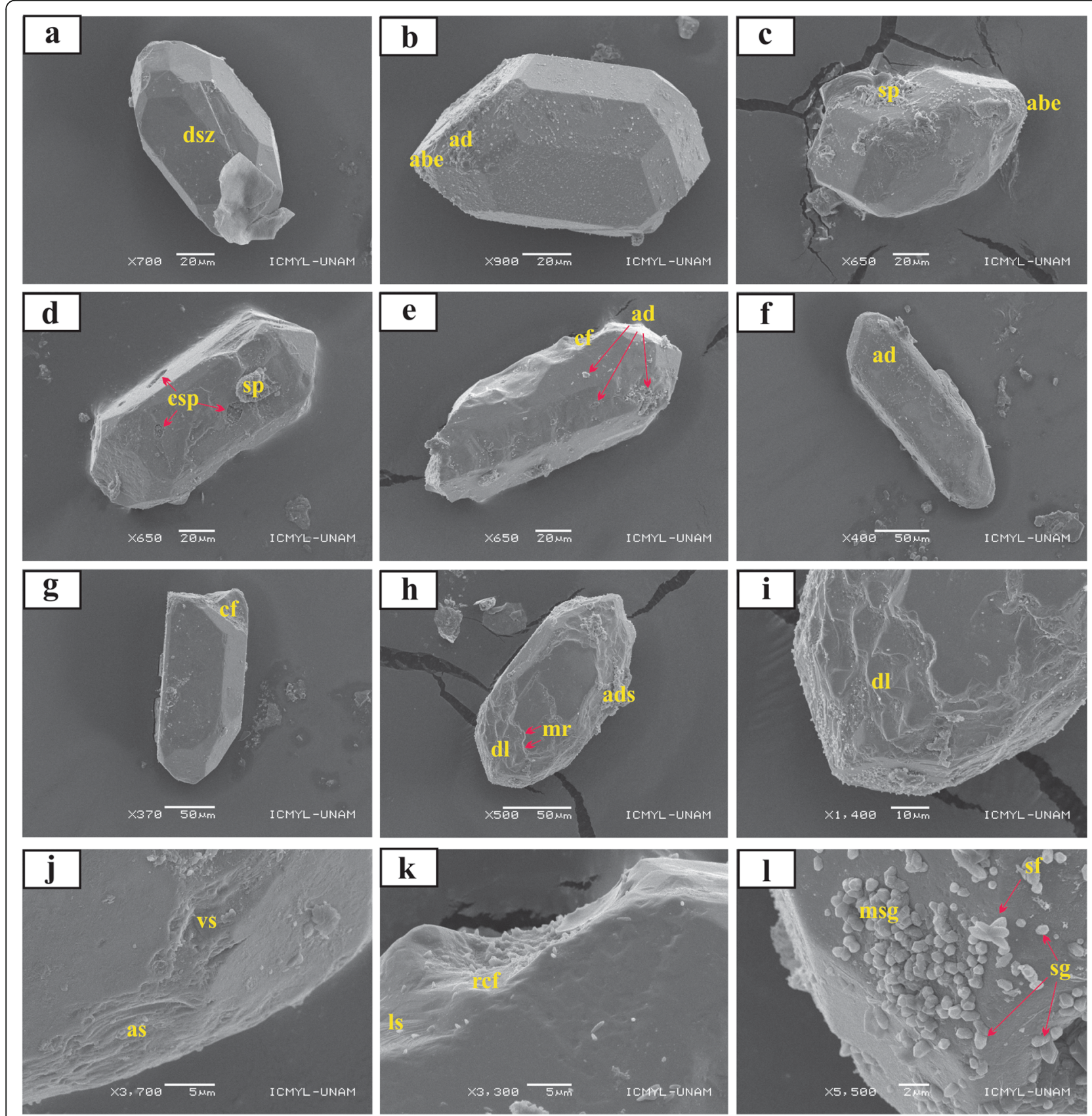

Fig. 7 Scanning Electron Microscopy (SEM) images showing surface microtextures of detrital zircons from the Palma Sola beach sediments. a Dual striated zircon (dsz) well abraded at both ends; b Zircon grain with an abraded edge (abe) and adhered particles (ad); c Zircon grain with an abraded edge (abe) and silica pellicle (sp); d Angular grain with circular solution pits (csp) on fracture plane and silica pellicle (sp); e Collision fracture (cf) with adhered particles (ad); $\mathbf{f}$ A sub-rounded zircon grain with adhered particles (ad); $\mathbf{g}$ A euhedral zircon grain with collision fracture (cf); $\mathbf{h}$ Zircon grain showing delamination (dl), meandering ridge (mr), and adhered particle appears to be silica globule (ads); $\mathbf{i}$ Enlarged view of grain $\mathbf{h}$ showing delamination (dl); $\mathbf{j}$ V-shaped percussion cracks (vs) and arc-shaped steps (as); $\mathbf{k}$ Reworked conchoidal fracture (rcf) with linear steps (Is); I Zircon grain with adhered particles (ad), silica globule ( $\mathrm{sg}$ ), merged silica globule (msg), and silica flower (sf), due to precipitation 
Table 2 Major element concentrations (in wt.\%) of the Riachuelos and Palma Sola beach sediments

\begin{tabular}{|c|c|c|c|c|c|c|c|c|c|c|c|c|c|}
\hline \multirow{2}{*}{$\begin{array}{l}\text { Beach } \\
\text { Sample }\end{array}$} & \multicolumn{13}{|c|}{ Riachuelos } \\
\hline & RIA1 & RIA2 & RIA3 & RIA4 & RIA5 & RIA6 & RIA7 & RIA8 & RIA9 & RIA10 & RIA11 & RIA12 & RIA13 \\
\hline $\mathrm{SiO}_{2}$ & 63.6 & 60.2 & 61.4 & 58.1 & 55.9 & 62.3 & 63.4 & 59.5 & 58.8 & 60.1 & 58.0 & 60.5 & 57.7 \\
\hline $\mathrm{TiO}_{2}$ & 0.96 & 1.07 & 0.67 & 0.90 & 0.82 & 1.03 & 1.04 & 0.76 & 1.09 & 1.06 & 1.13 & 0.72 & 0.96 \\
\hline $\mathrm{Al}_{2} \mathrm{O}_{3}$ & 14.4 & 13.9 & 13.5 & 14.1 & 12.2 & 14.2 & 15.4 & 14.2 & 14.9 & 13.3 & 11.1 & 15.7 & 12.4 \\
\hline $\mathrm{Fe}_{2} \mathrm{O}_{3}{ }^{\mathrm{a}}$ & 5.93 & 6.71 & 5.71 & 5.90 & 6.23 & 6.52 & 6.19 & 6.73 & 6.80 & 7.01 & 8.09 & 6.40 & 6.75 \\
\hline $\mathrm{MnO}$ & 0.08 & 0.10 & 0.05 & 0.09 & 0.10 & 0.09 & 0.08 & 0.06 & 0.08 & 0.10 & 0.13 & 0.06 & 0.10 \\
\hline $\mathrm{MgO}$ & 2.58 & 3.13 & 3.43 & 2.67 & 3.46 & 3.07 & 2.22 & 2.44 & 3.70 & 3.48 & 5.27 & 2.23 & 3.32 \\
\hline $\mathrm{CaO}$ & 5.09 & 6.19 & 4.85 & 7.64 & 9.96 & 5.57 & 4.75 & 4.96 & 4.59 & 6.55 & 7.95 & 5.95 & 7.86 \\
\hline $\mathrm{Na}_{2} \mathrm{O}$ & 3.07 & 2.88 & 2.91 & 2.69 & 2.19 & 2.92 & 3.16 & 2.72 & 2.95 & 2.80 & 2.08 & 3.17 & 2.67 \\
\hline $\mathrm{K}_{2} \mathrm{O}$ & 2.10 & 1.90 & 1.90 & 1.75 & 1.45 & 1.97 & 2.26 & 2.23 & 2.20 & 1.77 & 1.33 & 1.84 & 1.69 \\
\hline $\mathrm{P}_{2} \mathrm{O}_{5}$ & 0.20 & 0.23 & 0.05 & 0.24 & 0.24 & 0.20 & 0.12 & 0.05 & 0.04 & 0.25 & 0.23 & 0.04 & 0.24 \\
\hline LOI & 1.12 & 2.76 & 4.94 & 4.29 & 6.16 & 1.08 & 0.98 & 5.88 & 4.65 & 2.54 & 2.78 & 3.01 & 5.62 \\
\hline Sum & 99.1 & 99.2 & 99.5 & 98.4 & 98.7 & 98.9 & 99.5 & 99.6 & 99.8 & 98.9 & 98.1 & 99.6 & 99.3 \\
\hline CIX & 73.6 & 74.5 & 73.8 & 76.1 & 77.0 & 74.3 & 74.0 & 74.1 & 74.3 & 74.4 & 74.3 & 75.9 & 74.1 \\
\hline Beach & \multicolumn{4}{|c|}{ Riachuelos } & \multicolumn{9}{|c|}{ Palma Sola } \\
\hline Sample & RIA14 & RIA15 & \multicolumn{2}{|c|}{ Mean $\pm 1 s(n=15)$} & PAL1 & PAL2 & PAL3 & PAL4 & PAL5 & PAL6 & PAL7 & PAL8 & PAL9 \\
\hline $\mathrm{SiO}_{2}$ & 55.8 & 58.9 & \multicolumn{2}{|c|}{$59.6 \pm 2.38$} & 80.5 & 77.6 & 76.4 & 76.1 & 74.4 & 78.4 & 78.5 & 80.5 & 76.5 \\
\hline $\mathrm{TiO}_{2}$ & 1.37 & 1.12 & \multicolumn{2}{|c|}{$0.98 \pm 0.18$} & 0.39 & 0.49 & 0.56 & 0.54 & 0.77 & 0.46 & 0.61 & 0.48 & 0.58 \\
\hline $\mathrm{Al}_{2} \mathrm{O}_{3}$ & 12.9 & 14.6 & \multicolumn{2}{|c|}{$13.80 \pm 1.26$} & 9.21 & 9.98 & 10.3 & 9.96 & 10.1 & 9.87 & 7.05 & 8.20 & 10.2 \\
\hline $\mathrm{Fe}_{2} \mathrm{O}_{3}{ }^{\mathrm{a}}$ & 9.19 & 6.67 & \multicolumn{2}{|c|}{$6.72 \pm 0.89$} & 2.77 & 3.20 & 3.82 & 3.59 & 4.76 & 3.00 & 4.10 & 3.21 & 3.73 \\
\hline $\mathrm{MnO}$ & 0.15 & 0.09 & \multicolumn{2}{|c|}{$0.09 \pm 0.02$} & 0.03 & 0.04 & 0.04 & 0.04 & 0.05 & 0.03 & 0.06 & 0.04 & 0.04 \\
\hline $\mathrm{MgO}$ & 5.57 & 2.74 & \multicolumn{2}{|c|}{$3.29 \pm 0.99$} & 0.87 & 1.06 & 1.37 & 1.46 & 1.74 & 0.85 & 2.43 & 1.24 & 1.13 \\
\hline $\mathrm{CaO}$ & 8.24 & 5.58 & \multicolumn{2}{|c|}{$6.38 \pm 1.60$} & 1.97 & 2.31 & 2.65 & 2.78 & 3.03 & 2.22 & 3.47 & 2.31 & 2.48 \\
\hline $\mathrm{Na}_{2} \mathrm{O}$ & 2.54 & 2.96 & \multicolumn{2}{|c|}{$2.78 \pm 0.32$} & 1.90 & 1.98 & 2.12 & 2.04 & 2.02 & 2.04 & 1.37 & 1.59 & 1.99 \\
\hline $\mathrm{K}_{2} \mathrm{O}$ & 1.55 & 2.07 & \multicolumn{2}{|c|}{$1.87 \pm 0.28$} & 1.73 & 1.68 & 1.63 & 1.57 & 1.59 & 1.74 & 1.19 & 1.52 & 1.68 \\
\hline $\mathrm{P}_{2} \mathrm{O}_{5}$ & 0.28 & 0.04 & \multicolumn{2}{|c|}{$0.16 \pm 0.09$} & 0.03 & 0.02 & 0.06 & 0.03 & 0.05 & 0.02 & 0.03 & 0.04 & 0.03 \\
\hline LOI & 1.72 & 4.61 & \multicolumn{2}{|c|}{$3.48 \pm 1.81$} & 0.89 & 1.04 & 1.11 & 1.13 & 1.13 & 1.05 & 0.62 & 0.73 & 1.08 \\
\hline Sum & 99.3 & 99.3 & \multicolumn{2}{|c|}{$99.10 \pm 0.48$} & 100.3 & 99.4 & 100.1 & 99.2 & 99.6 & 99.6 & 99.4 & 99.9 & 99.4 \\
\hline $\mathrm{CIX}$ & 75.9 & 74.3 & \multicolumn{2}{|c|}{$74.8 \pm 1.0$} & 71.7 & 73.1 & 73.4 & 73.4 & 73.8 & 72.3 & 73.3 & 72.6 & 73.6 \\
\hline Beach & \multicolumn{13}{|c|}{ Palma Sola } \\
\hline Sample & PAL10 & PAL11 & PAL1 & PAL13 & PAL14 & PAL15 & PAL16 & PAL17 & PAL18 & PAL19 & PAL20 & \multicolumn{2}{|c|}{ Mean $\pm 1 s(n=20)$} \\
\hline $\mathrm{SiO}_{2}$ & 74.0 & 74.9 & 78.9 & 77.7 & 70.8 & 71.9 & 79.0 & 77.6 & 78.0 & 79.8 & 75.7 & $76.9 \pm$ & \\
\hline $\mathrm{TiO}_{2}$ & 0.59 & 0.54 & 0.48 & 0.87 & 0.58 & 0.42 & 0.66 & 0.42 & 0.72 & 0.55 & 0.51 & $0.56 \pm$ & \\
\hline $\mathrm{Al}_{2} \mathrm{O}_{3}$ & 10.6 & 9.92 & 9.18 & 7.70 & 8.05 & 7.89 & 8.25 & 7.32 & 8.82 & 8.28 & 9.07 & $9.00 \pm$ & \\
\hline $\mathrm{Fe}_{2} \mathrm{O}_{3}{ }^{\mathrm{a}}$ & 4.06 & 3.84 & 3.14 & 5.02 & 4.04 & 3.17 & 3.93 & 3.16 & 4.25 & 3.49 & 3.59 & $2.69 \pm$ & \\
\hline $\mathrm{MnO}$ & 0.05 & 0.05 & 0.04 & 0.06 & 0.06 & 0.05 & 0.05 & 0.04 & 0.05 & 0.04 & 0.05 & $0.04 \pm$ & \\
\hline $\mathrm{MgO}$ & 1.40 & 1.53 & 1.05 & 2.03 & 1.90 & 1.75 & 1.41 & 1.56 & 1.45 & 1.36 & 1.53 & $1.46 \pm$ & \\
\hline $\mathrm{CaO}$ & 3.16 & 3.71 & 2.36 & 2.97 & 6.79 & 6.96 & 2.51 & 3.59 & 2.41 & 2.31 & 4.19 & $3.21 \pm$ & \\
\hline $\mathrm{Na}_{2} \mathrm{O}$ & 2.22 & 1.97 & 1.89 & 1.51 & 1.54 & 1.48 & 1.64 & 1.39 & 1.72 & 1.68 & 1.72 & $1.79 \pm$ & \\
\hline $\mathrm{K}_{2} \mathrm{O}$ & 1.63 & 1.55 & 1.63 & 1.29 & 1.28 & 1.25 & 1.46 & 1.28 & 1.50 & 1.49 & 1.47 & $1.51 \pm$ & \\
\hline $\mathrm{P}_{2} \mathrm{O}_{5}$ & 0.09 & 0.10 & 0.03 & 0.02 & 0.11 & 0.09 & 0.03 & 0.07 & 0.04 & 0.04 & 0.08 & $0.05 \pm$ & \\
\hline LOI & 1.51 & 1.92 & 0.98 & 0.66 & 4.14 & 4.57 & 0.77 & 1.74 & 0.99 & 0.67 & 2.30 & $1.45 \pm$ & \\
\hline Sum & 99.3 & 100.0 & 99.7 & 99.8 & 99.3 & 99.6 & 99.7 & 98.2 & 99.9 & 99.6 & 100.2 & $99.6 \pm$ & \\
\hline CIX & 73.4 & 73.8 & 72.3 & 73.3 & 74.1 & 74.2 & 72.7 & 73.2 & 73.2 & 72.4 & 74.1 & $73.2 \pm$ & \\
\hline
\end{tabular}

std. Standard deviation, $1 \mathrm{~s}$ One standard deviation, $\mathrm{LO}$ Loss on ignition, $n$ Total number of samples

a means Total Fe expressed as $\mathrm{Fe}_{2} \mathrm{O}_{3} ;$ Chemical Index: $\mathrm{CIX}=100 \times\left[\mathrm{Al}_{2} \mathrm{O}_{3} /\left(\mathrm{Al}_{2} \mathrm{O}_{3}+\mathrm{Na}_{2} \mathrm{O}+\mathrm{K}_{2} \mathrm{O}\right)\right]$ (referring to Nesbitt and Young 1982; Cullers 2000; Buggle et al. 2011; Garzanti et al. 2014) 
Table 3 Trace element concentrations (in ppm) of the Riachuelos and Palma Sola beach sediments

\begin{tabular}{|c|c|c|c|c|c|c|c|c|c|c|c|}
\hline \multirow{2}{*}{$\begin{array}{l}\text { Beach } \\
\text { Sample }\end{array}$} & \multicolumn{11}{|c|}{ Riachuelos } \\
\hline & RIA1 & RIA2 & RIA3 & RIA4 & RIA5 & RIA6 & RIA7 & RIA8 & RIA9 & RIA10 & RIA11 \\
\hline $\mathrm{Ba}$ & 607 & 558 & 461 & 522 & 480 & 635 & 751 & 476 & 527 & 543 & 440 \\
\hline Co & 15.1 & 16.86 & 14.6 & 14.9 & 16.0 & 17.3 & 14.3 & 15.6 & 14.8 & 18.7 & 24.2 \\
\hline $\mathrm{Cr}$ & 67.9 & 77.6 & 78.2 & 68.0 & 72.8 & 79.3 & 61.2 & 81.6 & 71.4 & 94.8 & 131.4 \\
\hline Cs & 1.99 & 1.53 & 1.42 & 0.6 & 0.64 & 1.93 & 2.28 & 1.58 & 1.78 & 1.71 & 1.36 \\
\hline $\mathrm{Cu}$ & 25.4 & 23.5 & 22.8 & 20.3 & 21.4 & 24.4 & 22.4 & 25 & 26.8 & 24.5 & 21.6 \\
\hline $\mathrm{Hf}$ & 4.69 & 4.21 & 3.78 & 3.47 & 3.03 & 4.77 & 4.91 & 4.69 & 3.31 & 4.16 & 3.65 \\
\hline Mo & 1.42 & 1.25 & 1.22 & 1.18 & 1.07 & 1.35 & 1.46 & 1.06 & 1.21 & 1.23 & 1.07 \\
\hline $\mathrm{Nb}$ & 17.9 & 17.2 & 16.4 & 15.3 & 13.6 & 18.7 & 20.6 & 16.9 & 17.6 & 17.1 & 14.2 \\
\hline $\mathrm{Ni}$ & 26.7 & 30.4 & 33.6 & 26.0 & 31.2 & 31.5 & 21.4 & 37.6 & 28.7 & 34.8 & 53.2 \\
\hline $\mathrm{Pb}$ & 13.1 & 10.7 & 11.6 & 10.46 & 9.49 & 11.7 & 13.1 & 12.7 & 9.83 & 10.77 & 8.93 \\
\hline $\mathrm{Rb}$ & 56.6 & 45.3 & 32.8 & 26.9 & 24.3 & 55.5 & 61.3 & 40.8 & 37.9 & 50.8 & 36.9 \\
\hline Sc & 12.7 & 12.81 & 13.6 & 7.91 & 8.35 & 19.2 & 21.8 & 15.9 & 18.6 & 14.95 & 22.7 \\
\hline $\mathrm{Sr}$ & 477 & 471 & 450 & 427 & 412 & 501 & 598 & 466 & 437 & 460 & 371 \\
\hline $\mathrm{Ta}$ & 5.84 & 5.47 & 4.86 & 4.95 & 4.3 & 6.15 & 8.89 & 5.38 & 4.69 & 6.11 & 4.6 \\
\hline Th & 7.56 & 6.34 & 6.12 & 4.22 & 2.9 & 7.52 & 12.4 & 5.68 & 6.47 & 6.61 & 5.56 \\
\hline U & 2.55 & 2.25 & 2.39 & 2.14 & 1.87 & 2.52 & 2.7 & 2.28 & 1.97 & 2.15 & 1.65 \\
\hline V & 117 & 132 & 136 & 120 & 117 & 134 & 126 & 125 & 139 & 144 & 170 \\
\hline Y & 12.3 & 12.8 & 10.6 & 8.75 & 8.2 & 12.9 & 18.7 & 9.67 & 11.9 & 14.8 & 18.5 \\
\hline$Z n$ & 59.0 & 59.0 & 52.6 & 53 & 53.5 & 60.7 & 67.4 & 54.8 & 58.7 & 71.1 & 79.2 \\
\hline $\mathrm{Zr}$ & 171 & 154 & 115 & 128 & 112 & 171 & 173 & 126 & 130 & 143 & 118 \\
\hline Th/U & 2.97 & 2.82 & 2.56 & 1.98 & 1.55 & 2.99 & 4.57 & 2.49 & 3.28 & 3.07 & 3.38 \\
\hline $\mathrm{Rb} / \mathrm{Sr}$ & 0.12 & 0.1 & 0.07 & 0.06 & 0.06 & 0.11 & 0.1 & 0.09 & 0.09 & 0.11 & 0.1 \\
\hline $\mathrm{Ba} / \mathrm{Sr}$ & 1.27 & 1.19 & 1.02 & 1.22 & 1.17 & 1.27 & 1.26 & 1.02 & 1.21 & 1.18 & 1.19 \\
\hline Beach & \multicolumn{5}{|c|}{ Riachuelos } & \multicolumn{6}{|c|}{ Palma Sola } \\
\hline Sample & RIA12 & RIA13 & RIA14 & RIA15 & Mean $\pm 1 \mathrm{~s}(n=15)$ & PAL1 & PAL2 & PAL3 & PAL4 & PAL5 & PAL6 \\
\hline $\mathrm{Ba}$ & 530 & 549 & 524 & 522 & $542 \pm 77$ & 482 & 368 & 414 & 412 & 488 & 380 \\
\hline Co & 17.9 & 18.0 & 26.9 & 19.7 & $17.70 \pm 3.63$ & 7.78 & 10.2 & 8.6 & 9.68 & 11.9 & 11.4 \\
\hline $\mathrm{Cr}$ & 68.3 & 86.6 & 148.5 & 70.6 & $83.9 \pm 24.5$ & 165 & 139 & 123 & 165 & 168 & 120 \\
\hline Cs & 1.48 & 1.66 & 1.46 & 1.69 & $1.54 \pm 0.45$ & 1.59 & 1.42 & 1.41 & 1.56 & 1.6 & 1.58 \\
\hline $\mathrm{Cu}$ & 23.0 & 20.9 & 26.0 & 26.0 & $23.60 \pm 2.04$ & 7.59 & 7.43 & 8.24 & 8.45 & 11.2 & 9.58 \\
\hline $\mathrm{Hf}$ & 3.26 & 3.95 & 4.82 & 4.06 & $4.05 \pm 0.63$ & 2.26 & 2.15 & 2.83 & 3.45 & 3.92 & 2.78 \\
\hline Mo & 1.31 & 1.18 & 1.24 & 1.16 & $1.23 \pm 0.12$ & 2.02 & 1.56 & 1.63 & 1.75 & 2.13 & 1.46 \\
\hline $\mathrm{Nb}$ & 19.7 & 15.87 & 18.3 & 18.5 & $17.20 \pm 1.92$ & 7.08 & 7.69 & 7.98 & 7.26 & 11.5 & 10.5 \\
\hline $\mathrm{Ni}$ & 29.4 & 33.23 & 60.0 & 34.46 & $34.1 \pm 10.0$ & 10.6 & 11.6 & 10.9 & 13.6 & 12.4 & 12.75 \\
\hline $\mathrm{Pb}$ & 10.2 & 10.52 & 9.9 & 12.6 & $11.00 \pm 1.35$ & 6.79 & 5.63 & 6.35 & 6.47 & 6.65 & 5.89 \\
\hline $\mathrm{Rb}$ & 29.5 & 46.6 & 41.4 & 44.6 & $42.1 \pm 11.1$ & 51.6 & 45.7 & 40.6 & 38.5 & 46.7 & 45.8 \\
\hline SC & 9.78 & 16.91 & 22.7 & 14.7 & $15.50 \pm 4.86$ & 4.94 & 5.12 & 5.36 & 10.3 & 10.9 & 9.7 \\
\hline Sr & 461 & 474 & 452 & 480 & $462 \pm 49$ & 243 & 232 & 234 & 280 & 279 & 204 \\
\hline $\mathrm{Ta}$ & 7.65 & 4.99 & 5.94 & 4.29 & $5.61 \pm 1.26$ & 0.45 & 0.44 & 0.52 & 0.51 & 0.66 & 0.58 \\
\hline Th & 8.63 & 6.84 & 7.06 & 7.61 & $6.77 \pm 2.09$ & 3.21 & 2.58 & 3.4 & 3.56 & 3.75 & 3.47 \\
\hline U & 2.17 & 2.16 & 2.16 & 2.46 & $2.23 \pm 0.27$ & 0.91 & 0.96 & 0.99 & 0.95 & 1.11 & 0.84 \\
\hline V & 159 & 137 & 196 & 129 & $139.0 \pm 21.6$ & 51.0 & 65.9 & 61.4 & 75.5 & 105 & 63.8 \\
\hline Y & 13.8 & 16.18 & 18.2 & 15.5 & $13.50 \pm 3.43$ & 7.89 & 9.84 & 8.39 & 11.5 & 10.7 & 10.6 \\
\hline
\end{tabular}


Table 3 Trace element concentrations (in ppm) of the Riachuelos and Palma Sola beach sediments (Continued)

\begin{tabular}{|c|c|c|c|c|c|c|c|c|c|c|c|}
\hline Zn & 61.8 & 65.5 & 81.4 & 55.7 & $62.2 \pm 9.1$ & 31 & 42.6 & 37.5 & 51.5 & 52.7 & 40.7 \\
\hline $\mathrm{Zr}$ & 158 & 141 & 160 & 120 & $141.0 \pm 21.8$ & 92.0 & 111 & 112 & 89.0 & 174 & 107 \\
\hline Th/U & 3.98 & 3.17 & 3.26 & 3.09 & $3.01 \pm 0.73$ & 3.55 & 2.7 & 3.43 & 3.76 & 3.37 & 4.12 \\
\hline $\mathrm{Rb} / \mathrm{Sr}$ & 0.06 & 0.1 & 0.09 & 0.09 & $0.09 \pm 0.01$ & 0.21 & 0.2 & 0.17 & 0.14 & 0.17 & 0.22 \\
\hline $\mathrm{Ba} / \mathrm{Sr}$ & 1.15 & 1.16 & 1.16 & 1.09 & $1.17 \pm 0.07$ & 1.99 & 1.59 & 1.76 & 1.47 & 1.75 & 1.87 \\
\hline Beach & \multicolumn{11}{|c|}{ Palma Sola } \\
\hline Sample & PAL7 & PAL8 & PAL9 & PAL10 & PAL11 & PAL13 & PAL15 & PAL17 & PAL20 & Mean $\pm 1 \mathrm{~s}(n=15)$ & \\
\hline $\mathrm{Ba}$ & 358 & 402 & 495 & 410 & 449 & 373 & 374 & 384 & 423 & $414.0 \pm 45.1$ & \\
\hline Co & 12.1 & 12.7 & 9.3 & 7.26 & 11.3 & 12.0 & 9.57 & 10.0 & 9.39 & $10.20 \pm 1.63$ & \\
\hline $\mathrm{Cr}$ & 195 & 160 & 158 & 135 & 161 & 197 & 108 & 161 & 159 & $154.0 \pm 25.2$ & \\
\hline Cs & 0.98 & 1.13 & 1.73 & 1.46 & 1.49 & 1.12 & 1.17 & 1.05 & 1.31 & $1.37 \pm 0.23$ & \\
\hline $\mathrm{Cu}$ & 8.15 & 7.69 & 9.93 & 8.64 & 9.66 & 9.81 & 7.71 & 7.5 & 7.79 & $8.62 \pm 1.14$ & \\
\hline $\mathrm{Hf}$ & 2.16 & 3.26 & 3.07 & 2.13 & 2.52 & 4.09 & 1.99 & 1.93 & 2.71 & $2.75 \pm 0.69$ & \\
\hline Mo & 2.27 & 2.06 & 1.86 & 1.76 & 2.21 & 1.95 & 1.36 & 2.17 & 2.18 & $1.89 \pm 0.29$ & \\
\hline $\mathrm{Nb}$ & 7.38 & 8.63 & 9.82 & 7.43 & 8.11 & 10.34 & 6.31 & 6.16 & 7.88 & $8.27 \pm 1.57$ & \\
\hline $\mathrm{Ni}$ & 13.02 & 11.7 & 11.2 & 10.5 & 12.2 & 12.4 & 11.5 & 11.6 & 11.3 & $11.80 \pm 0.89$ & \\
\hline $\mathrm{Pb}$ & 5.33 & 7.48 & 9.56 & 6.76 & 7.31 & 5.78 & 6.81 & 6.51 & 6.19 & $6.63 \pm 1.00$ & \\
\hline $\mathrm{Rb}$ & 33.9 & 38.7 & 51.4 & 41.9 & 44.1 & 36.4 & 37.3 & 37.3 & 43.2 & $42.20 \pm 5.36$ & \\
\hline Sc & 14.9 & 10.7 & 7.94 & 7.26 & 9.17 & 11.37 & 8.97 & 8.36 & 7.76 & $8.85 \pm 2.67$ & \\
\hline Sr & 189 & 206 & 277 & 211 & 274 & 193 & 259 & 207 & 216 & $234 \pm 33$ & \\
\hline Ta & 0.44 & 0.42 & 0.61 & 0.56 & 0.52 & 0.6 & 0.39 & 0.38 & 0.49 & $0.50 \pm 0.08$ & \\
\hline Th & 2.29 & 2.89 & 3.81 & 3.36 & 3.46 & 2.76 & 2.68 & 2.44 & 2.83 & $3.10 \pm 0.49$ & \\
\hline U & 0.66 & 0.85 & 1.1 & 1.06 & 1.09 & 0.85 & 0.93 & 0.8 & 0.84 & $0.93 \pm 0.13$ & \\
\hline V & 83.8 & 67.9 & 77.9 & 71.7 & 70.8 & 108 & 62.8 & 60.9 & 67.8 & $73.0 \pm 15.7$ & \\
\hline Y & 11.2 & 12.5 & 9.79 & 10.7 & 12.5 & 10.1 & 13.7 & 11.35 & 9.96 & $10.70 \pm 1.52$ & \\
\hline Zn & 39.7 & 38.5 & 41.8 & 51.5 & 52.3 & 45.4 & 39.8 & 39.2 & 37.2 & $42.70 \pm 6.53$ & \\
\hline $\mathrm{Zr}$ & 87 & 92 & 128 & 101 & 104 & 178 & 83.9 & 80.2 & 113 & $110.0 \pm 29.8$ & \\
\hline Th/U & 3.47 & 3.42 & 3.46 & 3.17 & 3.17 & 3.25 & 2.88 & 3.06 & 3.37 & $3.34 \pm 0.34$ & \\
\hline $\mathrm{Rb} / \mathrm{Sr}$ & 0.18 & 0.19 & 0.19 & 0.2 & 0.16 & 0.19 & 0.14 & 0.18 & 0.2 & $0.18 \pm 0.02$ & \\
\hline $\mathrm{Ba} / \mathrm{Sr}$ & 1.89 & 1.95 & 1.79 & 1.95 & 1.64 & 1.93 & 1.45 & 1.86 & 1.95 & $1.79 \pm 0.18$ & \\
\hline
\end{tabular}

std. Standard deviation, $1 \mathrm{~s}$ One standard deviation, $n$ Total number of samples

obtained from the nearby and distal major source terranes along the coastal regions of the Gulf of Mexico (such as Chiapas Massif Complex, Eastern Alkaline Province, Mesa Central Province, and Oaxacan Complex in Fig. 2) was conducted to investigate the provenance of the Riachuelos and Palma Sola beach sediments.

\section{Results}

\subsection{Mineralogy of bulk sediments}

$\mathrm{X}$-Ray diffraction analysis reveals that zircon, quartz, magnetite, and ilmenite are the major and chromite, geikielite, spinel, and labradorite are the minor minerals in the Riachuelos (Fig. 3a, b) and Palma Sola (Fig. 3c, d) beach sediments. Based on the quantitative compositional data (Additional File 1: Supplementary Information 1) obtained by SEM-EDS analysis, heavy minerals such as apatite
(Fig. 4a), zircon (Fig. 4b), and magnetite (Fig. 4c) are identified in Riachuelos beach sediments, and titanomagnetite (Fig. 4d), ilmenite (Fig. 4e), and quartz (Fig. 4f) are identified in Palma Sola beach sediments.

\subsection{Zircon $\mathrm{CL}$ images}

Zircons collected from the Riachuelos and Palma Sola beach sediments are mostly prismatic and showing oscillatory zoning with luminescent overgrowths, which supports for a magmatic origin (Fig. 5). More cathodoluminescence images for the zircon sequences of the Riachuelos (Supplementary Information 8) and Palma Sola (Supplementary Information 9) beach sediments are provided in Additional File 2 to better recognize their grain morphology. In general, the morphology differences between zircons from the 
Riachuelos and the Palma Sola beach sediments are not significant (Fig. 5).

\subsection{Zircon surface microtextures}

To infer the sedimentary provenance, transportation medium and depositional history of the beach sediments, microtextures on detrital zircon grains from the Riachuelos and Palma Sola beach sediments are investigated and interpreted based on the traditional classification procedures followed by various researchers (Mahaney 2002; Madhavaraju et al. 2009; Mahaney et al. 2012; ArmstrongAltrin and Natalhy-Pineda 2014; Vos et al. 2014; Mohammad et al. 2020). Microtextures of mechanical and chemical features identified on the Riachuelos and Palma Sola detrital zircons are listed in Table 1, and their SEM images are shown in Figs. 6 and 7, respectively.

The mechanical features identified in the Riachuelos zircons include well-abraded euhedral grains with broken edges (Fig. 6a, b, e, h, i), crescentic gouge (Fig. 6c, d), arc-shaped steps and linear steps (Fig. 6d, i), bulbous edge and reworked conchoidal fracture (Fig. 6f), and, collision fractures with straight groove (Fig. 6g, h, k). Similarly, the microtextures of chemical origin identified in the Riachuelos zircons are cavities, adhered particles (ad), solution and precipitation features $(\mathrm{s} / \mathrm{p})$, and silica pellicle (sp) (Fig. 6j-l).

The microtextures identified in the Palma Sola zircons are like the Riachuelos zircons which exhibit both mechanical and chemical features. The mechanical features include abraded edges (abe) (Fig. 7a), collision fractures (cf) (Fig. 7e, g), V-shaped percussion cracks (vs) and arcshaped steps (as) (Fig. 7j), meandering ridge (mr) (Fig. $7 \mathrm{~h}$ ), and reworked conchoidal fracture with linear steps (Fig. 7k). The chemical features include adhered particles (ad) (Fig. 7b, e, f), silica pellicle (sp) (Fig. 7c, d), circular solution pits (csp) (Fig. 7d), delamination (dl) (Fig. 7h, i), silica globule (sg) (Fig. 7h, l), and silica flower (sf) (Fig. 7l).

\subsection{Geochemical composition of bulk sediments}

The major element concentrations and their elemental ratios are provided in Table 2 (in wt.\%). The Riachuelos beach sediments are enriched in $\mathrm{Al}_{2} \mathrm{O}_{3}$ (the mean value of $14 \pm 1$ wt.\% with one-standard-deviation), $\mathrm{TiO}_{2}$ (mean $0.98 \pm 0.18$ wt.\%), $\mathrm{Fe}_{2} \mathrm{O}_{3}$ (mean $6.72 \pm 0.89$ wt.\%), and $\mathrm{P}_{2} \mathrm{O}_{5}$ (mean $0.16 \pm 0.09$ wt.\%) contents and depleted in $\mathrm{SiO}_{2}$ content (the mean value of $59.6 \pm 2.4 \mathrm{wt} . \%$ ) relative to the Palma Sola sediments (Table 3). Compared with the average upper continental crust (UCC; Taylor and McLennan 1985), the Riachuelos beach sediments are slightly enriched in $\mathrm{TiO}_{2}, \mathrm{Fe}_{2} \mathrm{O}_{3}, \mathrm{MnO}, \mathrm{MgO}$, and $\mathrm{CaO}$ contents, whereas $\mathrm{K}_{2} \mathrm{O}, \mathrm{Na}_{2} \mathrm{O}$, and $\mathrm{P}_{2} \mathrm{O}_{5}$ contents of both beach sediments are depleted (Fig. 8a).
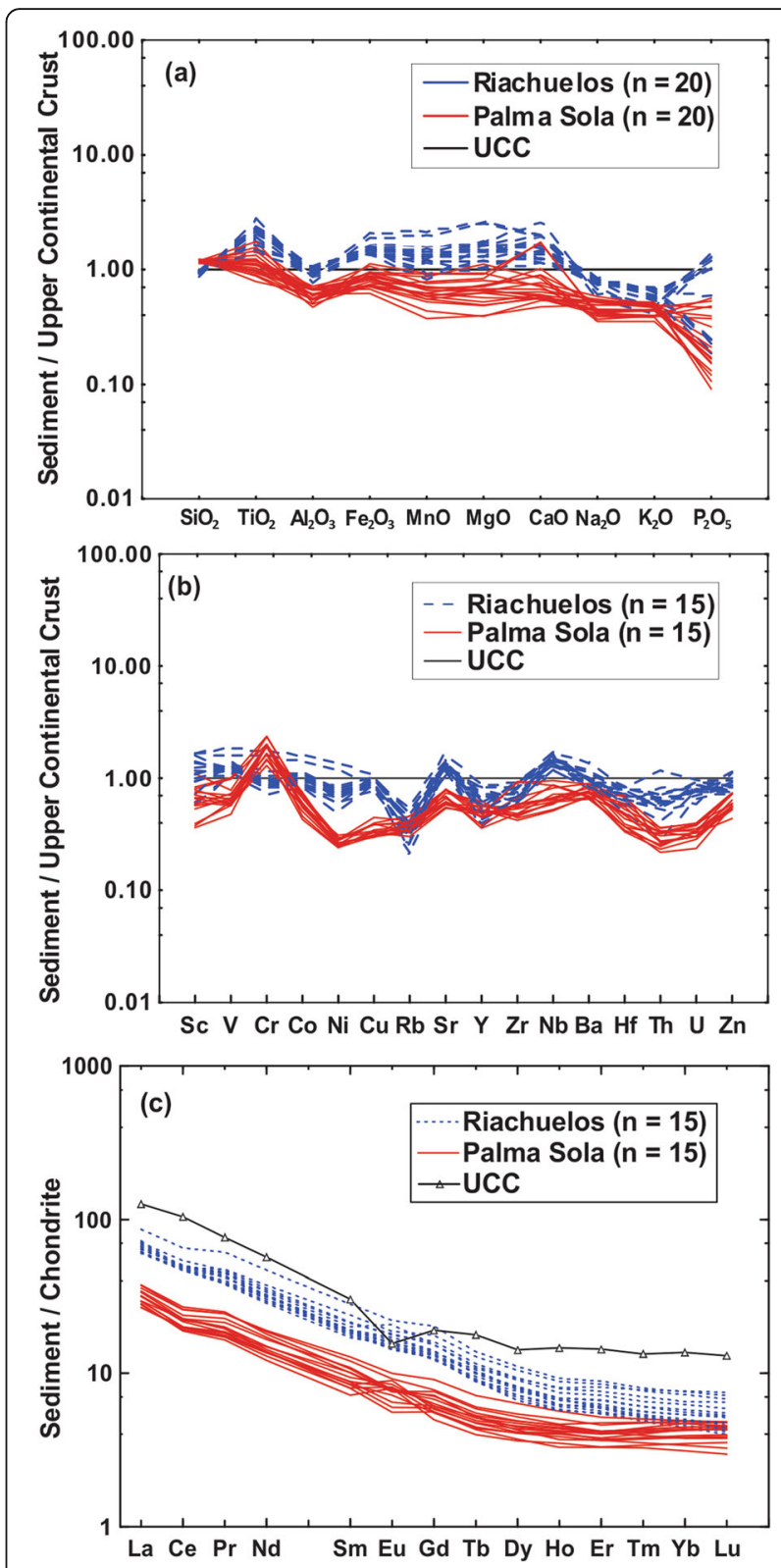

Fig. 8 Upper continental crust (UCC) and chondrite-normalized a major element, $\mathbf{b}$ trace element, and $\mathbf{c}$ rare earth element distribution patterns for the Riachuelos and Palma Sola beach sediments. Normalization values are from Taylor and McLennan (1985)

The trace element and REE concentrations of the Riachuelos and Palma Sola beach sediments are listed in Tables 3 and 4, respectively. Compared with average UCC values, the Riachuelos beach sediments are moderately enriched in $\mathrm{V}, \mathrm{Sr}, \mathrm{Nb}$, and $\mathrm{Ba}$ contents, whereas in Palma Sola beach sediments, other elements except $\mathrm{Cr}$ are moderately to strongly depleted (Fig. 8b). Among high field strength elements (HFSE), $\mathrm{Zr}$ and $\mathrm{Hf}$ are largely immobile during weathering and metamorphic processes (McLennan et al. 1993; Men et al. 2019). The 
Table 4 Rare earth element concentrations (in ppm) of the Riachuelos and Palma Sola beach sediments

\begin{tabular}{|c|c|c|c|c|c|c|c|c|c|c|c|}
\hline \multirow{2}{*}{$\begin{array}{l}\text { Beach } \\
\text { Sample }\end{array}$} & \multicolumn{11}{|c|}{ Riachuelos } \\
\hline & RIA1 & RIA2 & RIA3 & RIA4 & RIA5 & RIA6 & RIA7 & RIA8 & RIA9 & RIA10 & RIA11 \\
\hline La & 22.2 & 23.6 & 22.5 & 22.1 & 22.7 & 24.1 & 26.7 & 23.5 & 24.6 & 25.1 & 24.5 \\
\hline $\mathrm{Ce}$ & 46.2 & 47.3 & 45.6 & 44.6 & 45.1 & 48.2 & 46.2 & 47.9 & 46.5 & 45.5 & 46.6 \\
\hline $\operatorname{Pr}$ & 5.23 & 5.77 & 5.34 & 5.51 & 5.81 & 5.75 & 6.24 & 6.14 & 5.94 & 6.13 & 6.48 \\
\hline $\mathrm{Nd}$ & 20.3 & 22.8 & 20.9 & 21.6 & 23.1 & 22.8 & 24.0 & 22.8 & 21.6 & 24.6 & 26.7 \\
\hline Sm & 3.94 & 4.40 & 4.23 & 4.07 & 4.39 & 4.42 & 4.64 & 4.36 & 4.28 & 4.92 & 5.54 \\
\hline Eu & 1.35 & 1.44 & 1.25 & 1.27 & 1.26 & 1.49 & 1.81 & 1.38 & 1.28 & 1.57 & 1.63 \\
\hline Gd & 3.78 & 4.28 & 3.85 & 3.82 & 4.00 & 4.29 & 4.70 & 4.06 & 4.13 & 4.71 & 5.42 \\
\hline $\mathrm{Tb}$ & 0.52 & 0.58 & 0.51 & 0.51 & 0.53 & 0.58 & 0.64 & 0.59 & 0.52 & 0.62 & 0.75 \\
\hline Dy & 2.75 & 3.12 & 2.54 & 2.63 & 2.72 & 3.10 & 3.50 & 3.09 & 2.90 & 3.43 & 3.98 \\
\hline Ho & 0.53 & 0.58 & 0.49 & 0.49 & 0.52 & 0.59 & 0.69 & 0.53 & 0.57 & 0.63 & 0.75 \\
\hline $\mathrm{Er}$ & 1.51 & 1.66 & 1.38 & 1.35 & 1.41 & 1.67 & 2.02 & 1.47 & 1.51 & 1.79 & 2.12 \\
\hline $\mathrm{Tm}$ & 0.20 & 0.22 & 0.19 & 0.17 & 0.17 & 0.22 & 0.28 & 0.18 & 0.19 & 0.23 & 0.27 \\
\hline $\mathrm{Yb}$ & 1.33 & 1.38 & 1.21 & 1.13 & 1.09 & 1.43 & 1.90 & 1.18 & 1.19 & 1.55 & 1.80 \\
\hline Lu & 0.20 & 0.20 & 0.18 & 0.16 & 0.15 & 0.21 & 0.29 & 0.17 & 0.16 & 0.22 & 0.26 \\
\hline LREE & 98.0 & 104 & 98.0 & 98.0 & 101 & 105 & 108 & 105 & 103 & 106 & 110 \\
\hline HREE & 10.8 & 12.0 & 10.3 & 10.3 & 10.6 & 12.1 & 14.0 & 11.3 & 11.2 & 13.2 & 15.3 \\
\hline TREE & 110 & 117 & 110 & 109 & 113 & 119 & 124 & 117 & 115 & 121 & 127 \\
\hline $\mathrm{Eu} / \mathrm{Eu}^{*}$ & 1.05 & 1.00 & 0.93 & 0.97 & 0.90 & 1.03 & 1.17 & 0.99 & 0.92 & 0.99 & 0.90 \\
\hline Beach & \multicolumn{5}{|c|}{ Riachuelos } & \multicolumn{6}{|c|}{ Palma Sola } \\
\hline Sample & RIA12 & RIA13 & RIA14 & RIA15 & Mean $\pm 1 s(n=15)$ & PAL1 & PAL2 & PAL3 & PAL4 & PAL5 & PAL6 \\
\hline La & 25.6 & 25.9 & 31.8 & 26.1 & $24.70 \pm 2.45$ & 10.4 & 10.8 & 10.3 & 12.4 & 11.8 & 12.6 \\
\hline $\mathrm{Ce}$ & 44.6 & 51.5 & 62.6 & 45.7 & $47.60 \pm 4.51$ & 18.1 & 19.2 & 18.4 & 21.7 & 21.2 & 22.8 \\
\hline $\operatorname{Pr}$ & 5.24 & 6.39 & 8.41 & 5.34 & $5.98 \pm 0.79$ & 2.26 & 2.42 & 2.36 & 2.74 & 2.74 & 3.12 \\
\hline $\mathrm{Nd}$ & 22.4 & 25.5 & 33.4 & 22.8 & $23.7 \pm 3.2$ & 8.63 & 9.83 & 9.19 & 10.1 & 10.7 & 12.2 \\
\hline Sm & 4.42 & 4.98 & 6.51 & 4.18 & $4.62 \pm 0.66$ & 1.66 & 1.94 & 1.85 & 2.02 & 2.17 & 2.32 \\
\hline Eu & 1.37 & 1.54 & 1.93 & 1.37 & $1.46 \pm 0.20$ & 0.69 & 0.52 & 0.71 & 0.56 & 0.74 & 0.66 \\
\hline $\mathrm{Gd}$ & 3.74 & 4.92 & 6.23 & 4.21 & $4.41 \pm 0.69$ & 1.52 & 1.82 & 1.71 & 1.87 & 1.87 & 2.20 \\
\hline $\mathrm{Tb}$ & 0.55 & 0.67 & 0.8 & 0.61 & $0.60 \pm 0.09$ & 0.23 & 0.27 & 0.26 & 0.3 & 0.28 & 0.30 \\
\hline Dy & 3.02 & 3.54 & 4.23 & 2.96 & $3.17 \pm 0.49$ & 1.38 & 1.57 & 1.54 & 1.69 & 1.73 & 1.62 \\
\hline Ho & 0.58 & 0.68 & 0.79 & 0.57 & $0.60 \pm 0.09$ & 0.30 & 0.35 & 0.33 & 0.36 & 0.37 & 0.31 \\
\hline Er & 1.47 & 1.9 & 2.21 & 1.56 & $1.67 \pm 0.28$ & 0.82 & 0.92 & 0.91 & 1.02 & 1.02 & 0.91 \\
\hline $\mathrm{Tm}$ & 0.19 & 0.25 & 0.28 & 0.2 & $0.22 \pm 0.04$ & 0.11 & 0.11 & 0.12 & 0.12 & 0.13 & 0.13 \\
\hline $\mathrm{Yb}$ & 1.24 & 1.62 & 1.88 & 1.32 & $1.42 \pm 0.27$ & 0.86 & 0.97 & 0.95 & 0.93 & 1.17 & 0.84 \\
\hline Lu & 0.18 & 0.25 & 0.27 & 0.2 & $0.21 \pm 0.04$ & 0.13 & 0.15 & 0.15 & 0.14 & 0.18 & 0.12 \\
\hline LREE & 102 & 114 & 143 & 104 & $107.0 \pm 10.9$ & 41.0 & 44.2 & 42.1 & 49.0 & 48.6 & 53.1 \\
\hline HREE & 11.0 & 13.8 & 16.7 & 11.6 & $12.30 \pm 1.93$ & 5.36 & 6.17 & 5.96 & 6.44 & 6.76 & 6.43 \\
\hline TREE & 115 & 130 & 161 & 117 & $120.0 \pm 12.8$ & 47.1 & 50.9 & 48.7 & 56.0 & 56.1 & 60.2 \\
\hline $\mathrm{Eu} / \mathrm{Eu}^{*}$ & 1.00 & 0.94 & 0.91 & 0.99 & $0.98 \pm 0.07$ & 1.31 & 0.83 & 1.19 & 0.87 & 1.09 & 0.88 \\
\hline Beach & \multicolumn{11}{|c|}{ Palma Sola } \\
\hline Sample & PAL7 & PAL8 & PAL9 & PAL10 & PAL11 & PAL13 & PAL15 & PAL17 & PAL20 & Mean $\pm 1 s(n=15)$ & \\
\hline La & 9.90 & 10.9 & 11.6 & 13.8 & 13.7 & 9.85 & 13.2 & 11.6 & 10.8 & $11.60 \pm 1.32$ & \\
\hline $\mathrm{Ce}$ & 19.1 & 21.7 & 20.8 & 25.9 & 25.0 & 18.9 & 24.8 & 21.7 & 19.2 & $21.20 \pm 2.51$ & \\
\hline $\operatorname{Pr}$ & 2.55 & 2.68 & 2.54 & 3.44 & 3.36 & 2.38 & 3.35 & 2.94 & 2.50 & $2.76 \pm 0.39$ & \\
\hline
\end{tabular}


Table 4 Rare earth element concentrations (in ppm) of the Riachuelos and Palma Sola beach sediments (Continued)

\begin{tabular}{|c|c|c|c|c|c|c|c|c|c|c|}
\hline $\mathrm{Nd}$ & 10.5 & 9.83 & 9.72 & 12.8 & 13.2 & 9.65 & 13.5 & 11.8 & 9.73 & $10.80 \pm 1.55$ \\
\hline Sm & 2.34 & 1.84 & 1.95 & 2.46 & 2.78 & 2.04 & 2.95 & 2.52 & 1.95 & $2.19 \pm 0.37$ \\
\hline $\mathrm{Eu}$ & 0.67 & 0.48 & 0.78 & 0.66 & 0.8 & 0.70 & 0.87 & 0.68 & 0.63 & $0.68 \pm 0.10$ \\
\hline $\mathrm{Gd}$ & 2.07 & 1.71 & 1.78 & 2.32 & 2.38 & 1.94 & 2.79 & 2.16 & 1.69 & $1.99 \pm 0.33$ \\
\hline Tb & 0.31 & 0.25 & 0.28 & 0.34 & 0.35 & 0.30 & 0.41 & 0.32 & 0.25 & $0.30 \pm 0.05$ \\
\hline Dy & 1.85 & 1.42 & 1.65 & 1.98 & 2.06 & 1.80 & 2.42 & 1.86 & 1.56 & $1.74 \pm 0.27$ \\
\hline Ho & 0.39 & 0.28 & 0.36 & 0.40 & 0.43 & 0.38 & 0.48 & 0.39 & 0.34 & $0.36 \pm 0.05$ \\
\hline Er & 1.04 & 0.82 & 1.00 & 1.18 & 1.14 & 1.04 & 1.29 & 1.02 & 0.94 & $1.01 \pm 0.13$ \\
\hline Tm & 0.13 & 0.12 & 0.11 & 0.12 & 0.13 & 0.14 & 0.13 & 0.12 & 0.11 & $0.12 \pm 0.02$ \\
\hline $\mathrm{Yb}$ & 1.11 & 0.77 & 1.05 & 1.16 & 1.21 & 1.10 & 1.22 & 1.07 & 1.06 & $1.03 \pm 0.14$ \\
\hline Lu & 0.17 & 0.11 & 0.16 & 0.17 & 0.18 & 0.17 & 0.18 & 0.16 & 0.17 & $0.16 \pm 0.02$ \\
\hline LREE & 44.5 & 46.9 & 46.7 & 58.4 & 58.1 & 42.8 & 57.8 & 50.6 & 44.2 & $49.00 \pm 5.93$ \\
\hline HREE & 7.07 & 5.48 & 6.38 & 7.73 & 7.89 & 6.86 & 8.92 & 7.10 & 6.12 & $6.71 \pm 0.94$ \\
\hline TREE & 52.2 & 52.8 & 53.8 & 66.8 & 66.8 & 50.4 & 67.6 & 58.4 & 50.9 & $55.90 \pm 6.74$ \\
\hline $\mathrm{Eu} / \mathrm{Eu}^{*}$ & 0.91 & 0.82 & 1.26 & 0.83 & 0.93 & 1.06 & 0.91 & 0.87 & 1.03 & $0.99 \pm 0.16$ \\
\hline
\end{tabular}

$1 \mathrm{~s}$ One standard deviation, HREE Heavy Rare Earth Elements, LREE Low Rare Earth Elements, TREE Total Rare Earth Elements, Eu/Eu ${ }^{*} \mathrm{Eu}_{\mathrm{CN}} /\left(\mathrm{Sm}_{\mathrm{CN}} \times \mathrm{Gd}_{\mathrm{CN}}\right)^{1 / 2}$ and Ce/ $\mathrm{Ce}^{*} \mathrm{Ce}_{\mathrm{CN}} /\left(\mathrm{La}_{\mathrm{CN}} \times \mathrm{Pr}_{\mathrm{CN}}\right)^{1 / 2}$, where $\mathrm{CN}_{\mathrm{N}}$ means the chondrite-normalized value (Taylor and McLennan 1985)

concentrations of HFSE are similar between the Riachuelos $(\sim 110-173 \mathrm{ppm})$ and Palma Sola $(\sim 80-178$ ppm) sediments. The average concentration of transition trace elements like Sc, V, Co, and Ni are higher in Riachuelos beach sediments $(16 \pm 5 \mathrm{ppm}, 139 \pm 22 \mathrm{ppm}$, $18 \pm 3 \mathrm{ppm}$, and $34 \pm 4 \mathrm{ppm}$, respectively) than in the Palma Sola beach sediments $(9 \pm 3 \mathrm{ppm}, 73 \pm 16 \mathrm{ppm}, 10 \pm 2 \mathrm{ppm}$, and $12 \pm 1 \mathrm{ppm}$, respectively) (Table 3$)$. The $\Sigma$ REE content is also higher in the Riachuelos beach sediments $(\sim 109$ $116 \mathrm{ppm})$ than in the Palma Sola beach sediments $(\sim 47-$ $68 \mathrm{ppm}$ ) (Table 4). The chondrite-normalized (CN) REE patterns for the Riachuelos and Palma Sola beach sediments are similar, enriched in LREE $\left(\mathrm{La}_{\mathrm{CN}} / \mathrm{Sm}_{\mathrm{CN}}=\sim 2.78-3.93\right.$ and $\sim 2.66-3.95$, respectively), depleted in HREE $\left(\mathrm{Gd}_{\mathrm{CN}} /\right.$ $\mathrm{Yb}_{\mathrm{CN}}=\sim 2.0-2.9$ and $\sim 1.3-2.1$, respectively), with low negative to positive $\mathrm{Eu}$ anomalies $\left(\mathrm{Eu} / \mathrm{Eu}^{*}=\sim 0.90-1.17\right.$ and $\sim 0.81-1.31$, respectively) (Fig. $8 \mathrm{c}$ ).

\subsection{Geochemical composition of detrital zircons}

The trace (Nb, Hf, Th, and $\mathrm{U}$ ) and REE concentrations of detrital zircons from the Riachuelos and Palma Sola beach sediments are listed in Additional File 1 (Supplementary Information 2 and 3, respectively).

The variation in $\mathrm{Y}$ content between the Riachuelos zircons $(\sim 221-9300 \mathrm{ppm}, \mathrm{n}=146)$ and the Palma Sola zircons $(\sim 266-9400 \mathrm{ppm}, \mathrm{n}=117)$ is not significant. However, the variations in Th and $U$ contents of the Palma Sola zircons ( 21-2900 ppm and 28-2290 ppm, respectively) are slightly higher than those of the Riachuelos zircons $\quad(\sim 16-2017 \mathrm{ppm} \quad$ and $\sim 31-1451$ $\mathrm{ppm})$. The $\mathrm{Th} / \mathrm{U}$ ratios of detrital zircons are generally used to differentiate igneous and/or metamorphic origins: Th/U ratio for zircons of igneous origin is $>0.3$ and for zircons of metamorphic origin is $<0.1$ (Rubatto 2002). The Th/U ratios of the Riachuelos and Palma Sola detrital zircons, respectively varying between 0.14 and $1.56(0.67 \pm 0.29, n=146)$ and varying between 0.13 and $2.27(0.68 \pm 0.36, n=117)$, are both consistent with a magmatic source (Fig. 9).

Variations in $\Sigma$ REE and HREE contents of the Riachuelos zircons $(\sim 215-2275 \mathrm{ppm}$ and $\sim 209-2132 \mathrm{ppm}$, respectively) and of the Palma Sola zircons $(\sim 256-2697$ ppm and $\sim 250-2608 \mathrm{ppm}$, respectively) are large. The REE patterns between the Riachuelos and Palma Sola zircons are similar, with depleted LREE and enriched $\operatorname{HREE}\left(\mathrm{Gd}_{\mathrm{CN}} / \mathrm{Yb}_{\mathrm{CN}}=2-3\right.$ and $1.3-2.1$, respectively), and mostly with pronounced negative $\mathrm{Eu}$ and positive $\mathrm{Ce}$ anomalies (Figs. 10 and 11, respectively). A few zircons

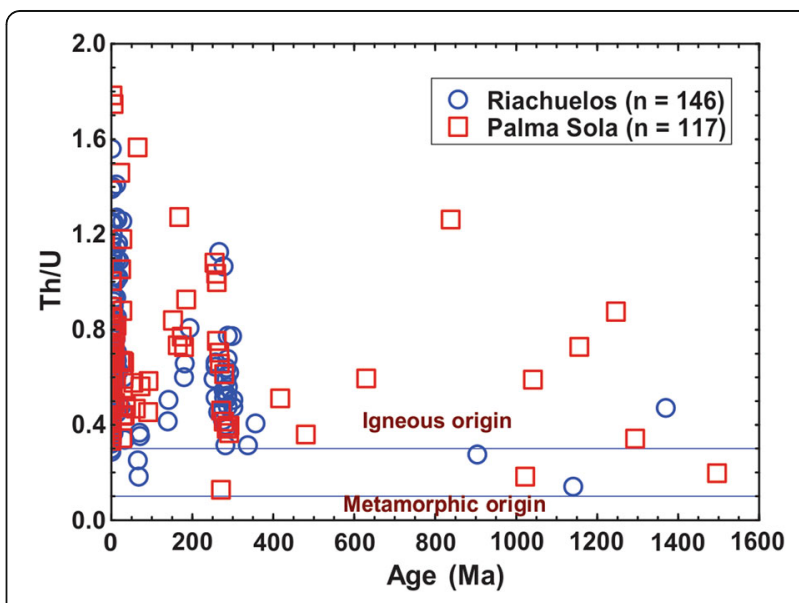

Fig. 9 Plot of Th/ $U$ ratios versus $U-P b$ ages for the Riachuelos and Palma Sola detrital zircons. Fields are after Rubatto (2002) 

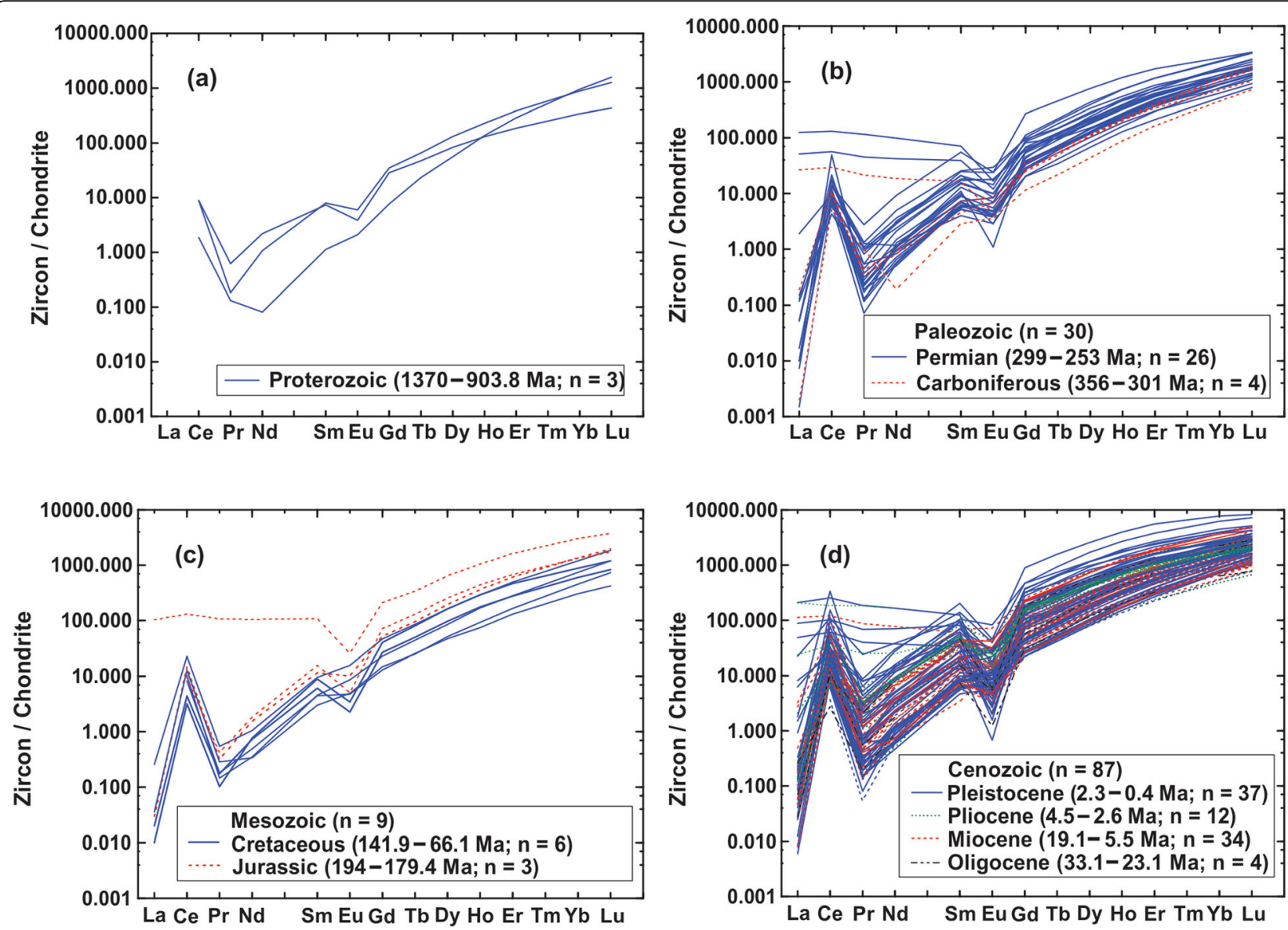

Fig. 10 Rare earth element patterns of the detrital zircons collected from the a Proterozoic $(n=3)$; b Paleozoic $(n=30)$; c Mesozoic $(n=9)$; $\mathbf{d}$ Cenozoic $(n=87)$ sediments in the Riachuelos beach area. Chondrite normalization values are from Taylor and McLennan (1985). $n=$ Number of zircon grains

enriched in LREE lack Ce anomalies. Moreover, the zircon REE patterns for the Proterozoic, Paleozoic, Mesozoic, and Cenozoic sediments in the Riachuelos (Fig. 10) and Palma Sola (Fig. 11) beach areas are similar.

\subsection{Detrital zircon $\mathrm{U}-\mathrm{Pb}$ dating ages}

Concordia plots and age probability density histograms of the Riachuelos and Palma Sola detrital zircons are shown in Fig. 12. In total, $146 \mathrm{U}-\mathrm{Pb}$ ages for the detrital zircons from the Riachuelos beach sediments are available and 129 involved provide the concordant ages (Fig. 12a, b; Additional File 1: Supplementary Information 4). Two significant age populations are identified as: (1) the Paleozoic $(\sim 356.5-252.5 \mathrm{Ma} ; n=30)$, including four Carboniferous ages and 26 Permian ages; and, (2) the Cenozoic ( 33.1-0.4 Ma; $n=87$ ), including four Oligocene ages, 34 Miocene ages, 12 Pliocene ages and 37 Pleistocene ages. In addition, nine zircon $\mathrm{U}-\mathrm{Pb}$ ages of $\sim 194-66 \mathrm{Ma}$ represent the Mesozoic, among which, three are of Early Jurassic ages $(\sim 194-179 \mathrm{Ma})$ and six are of Cretaceous ( 141.9-66.1 $\mathrm{Ma}$ ); and three $\mathrm{U}-\mathrm{Pb}$ ages of $\sim 1370-903.8 \mathrm{Ma}$ represent the Proterozoic Period.
In total, 117 detrital zircons from the Palma Sola beach sediments are analysed and 102 spots provide concordant ages (Fig. 12c, d; Additional File 1: Supplementary Information 5). The Palma Sola zircons exhibit two prominent age peaks of the Paleozoic $(\sim 480-255$ $\mathrm{Ma} ; n=15)$ and the Cenozoic $(\sim 64.9-0.4 \mathrm{Ma} ; n=69)$, like the Riachuelos zircon ages. Besides, minor age peaks are concentrated in the Proterozoic $(\sim 1652-630 \mathrm{Ma}$; $n=9)$ and the Mesozoic $(\sim 185.5-72.4 \mathrm{Ma} ; n=9)$ (Fig. 12c, d).

\section{Discussion}

\subsection{Statistical analysis of geochemistry data}

\subsubsection{Pearson's correlation coefficient}

The correlation technique is applied in this study to measure the magnitude and direction of the association of elements between the Riachuelos and Palma Sola beach sediments. The correlation of $\mathrm{SiO}_{2}$ versus $\mathrm{Al}_{2} \mathrm{O}_{3}$, $\mathrm{Fe}_{2} \mathrm{O}_{3}, \mathrm{MnO}, \mathrm{CaO}, \mathrm{MgO}$, and $\mathrm{TiO}_{2}$ is statistically not significant for the Riachuelos $(r=0.39,-0.83,-0.80$, $0.72,-0.73$, and -0.62 , respectively; $n=15$ ) and Palma Sola $(r=-0.53,-0.54,-0.38,-0.50,-0.29$, and -0.40 , 

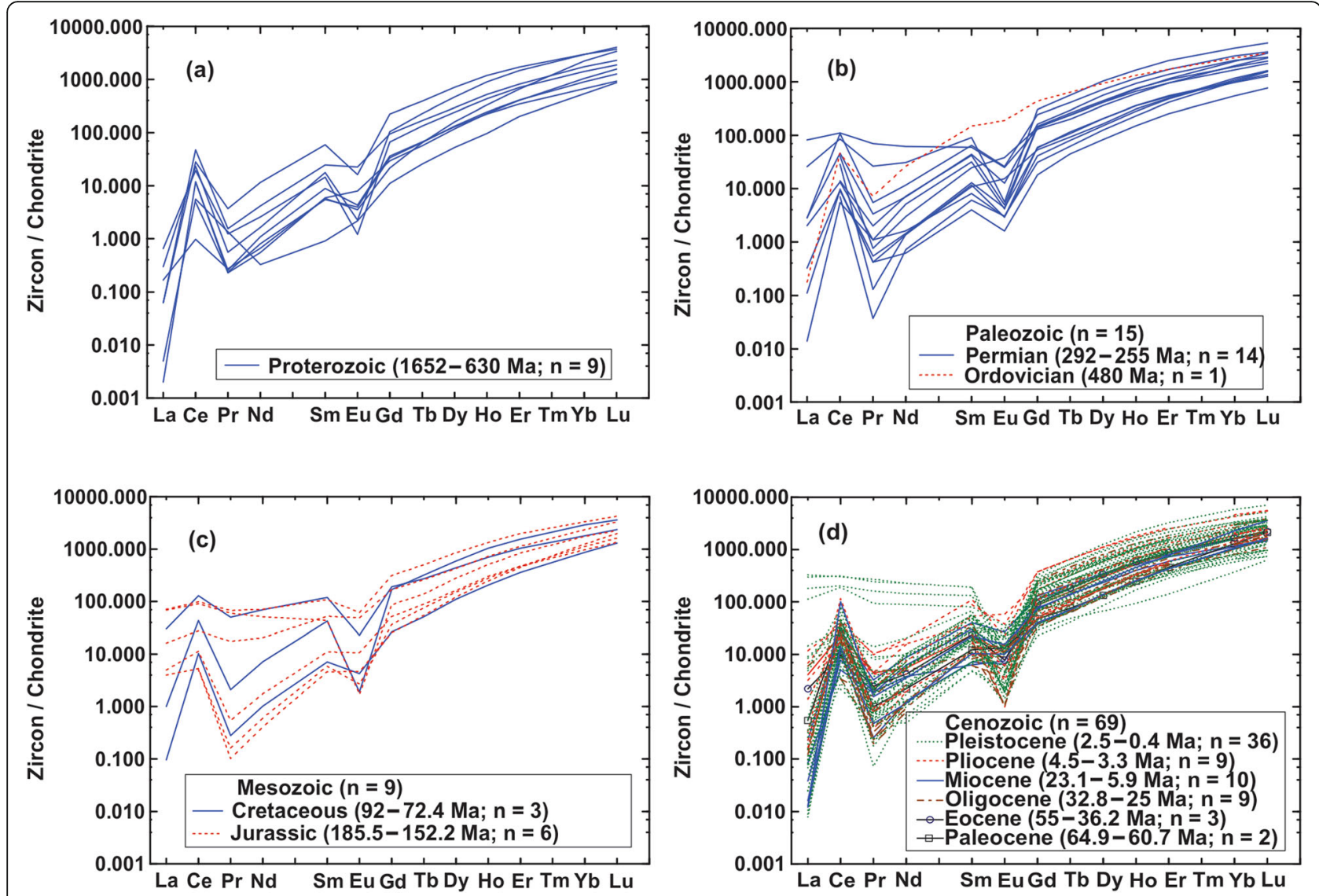

Fig. 11 Rare earth element patterns of the detrital zircons collected from the a Proterozoic $(n=9)$; b Paleozoic $(n=15) ; \mathbf{c}$ Mesozoic $(n=9) ; \mathbf{d}$ Cenozoic $(n=69)$ sediments in the Palma Sola beach area. Chondrite normalization values are from Taylor and McLennan (1985). $n=$ Number of zircon grains

respectively; $n=20$ ) beach sediments (critical $t$ value for 99\% confident level is 0.537; Verma 2005), reflecting the quartz abundance effect. For the Riachuelos beach sediments, $\mathrm{SiO}_{2}$ exhibits a significant positive correlation with $\mathrm{K}_{2} \mathrm{O}$ and $\mathrm{Na}_{2} \mathrm{O}$ ( $r=0.66$ and 0.63 , respectively), whereas this correlation is not significant for the Palma Sola sediments $(r=-0.08$ and -0.47 , respectively), which is probably due to variations in the relative proportions of K-feldspar and plagioclase, respectively (Bhattacharjee et al. 2018; Madhavaraju et al. 2020). This is also revealed in differences in $\mathrm{K}_{2} \mathrm{O}$ and $\mathrm{Na}_{2} \mathrm{O}$ contents between the Riachuelos ( 1.33-2.26 wt.\% and 2.083.17 wt.\%, respectively) and Palma Sola $(\sim 1.19-1.74$ wt.\% and $\sim 1.37-2.22$ wt.\%, respectively) beach sediments (Table 2). A significant positive correlation between $\mathrm{Al}_{2} \mathrm{O}_{3}$ and $\mathrm{K}_{2} \mathrm{O}$ for the Riachuelos $(r=0.81)$ and Palma Sola $(r=0.87)$ beach sediments indicates that a considerable proportion of $\mathrm{K}$ is associated with fine-grained sediments or phyllosilicates (Etemad-Saeed et al. 2015; Greggio et al. 2018; Udayanapillai et al. 2020). $\mathrm{Al}_{2} \mathrm{O}_{3}$ against $\mathrm{TiO}_{2}$ reveals a negative correlation for the Riachuelos $(r=-0.36, n=15)$ and Palma Sola $(r=-0.01$, $n=20$ ) beach sediments, indicating that $\mathrm{TiO}_{2}$ is not associated with aluminous clays or its association with titano-magnetite and ilmenite (Nagarajan et al. 2015, 2017; Papadopoulos et al. 2019). Similarly, a weak correlation between $\mathrm{Al}_{2} \mathrm{O}_{3}$ and $\mathrm{P}_{2} \mathrm{O}_{5}$ for the Riachuelos $(r=-0.70, n=15)$ and Palma Sola $(r=0.13, n=$ 20) beach sediments suggests $\mathrm{P}_{2} \mathrm{O}_{5}$ content is associated with detrital minerals, probably apatite (Fig. 4a).

The correlation between $\mathrm{Al}_{2} \mathrm{O}_{3}$ and HFSE like $\mathrm{Zr}, \mathrm{Y}$, and $\mathrm{Hf}$ is statistically not significant for the Riachuelos $(r=0.29,-0.28$, and 0.05 , respectively; $n=15)$ and Palma Sola $(r=0.17,-0.29$, and 0.12 , respectively; $n=15)$ beach sediments, which indicates that HFSEs are not associated with phyllosilicates but with accessory minerals. Similarly, $\mathrm{Sc}, \mathrm{V}, \mathrm{Co}, \mathrm{Cr}$, and $\mathrm{Ni}$ are all negatively correlated with $\mathrm{Al}_{2} \mathrm{O}_{3}$ for the Riachuelos $(r=-0.31,-0.40,-0.60,-0.73$, and -0.67 , respectively; $n=15$ ) and Palma Sola beach sediments $(r=-0.51,-0.09,-0.42,-0.45$, and -0.19 , respectively; $n=15$ ), suggesting the association of these trace elements with accessory components.

The correlation of $\Sigma$ REE with $\mathrm{SiO}_{2}, \mathrm{Al}_{2} \mathrm{O}_{3}, \mathrm{~K}_{2} \mathrm{O}$, and $\mathrm{Na}_{2} \mathrm{O}$ is statistically not significant for the Riachuelos 


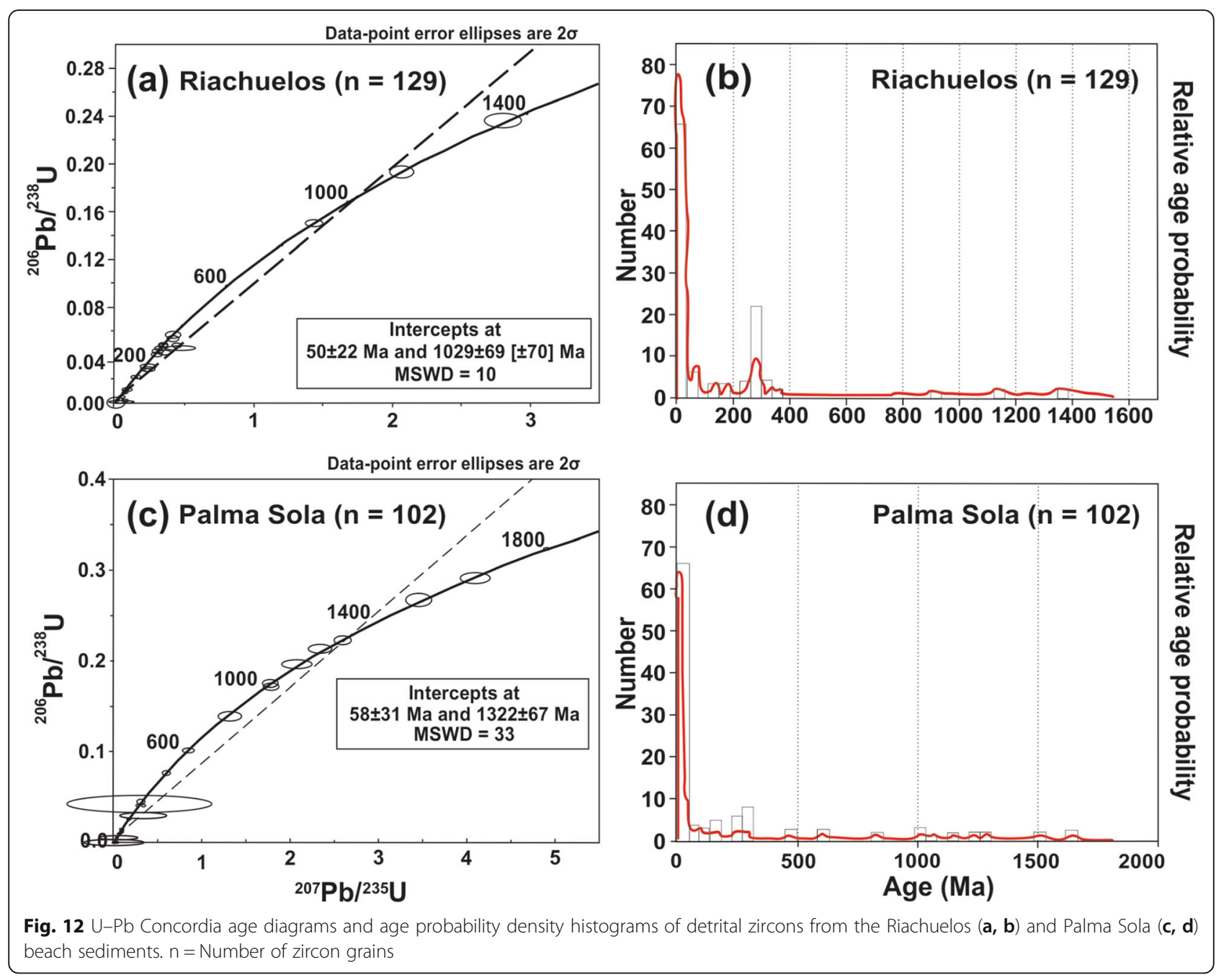

$(r=-0.73,-0.44,-0.41$, and -0.37 , respectively) and Palma Sola $(r=-0.60,0.20,-0.07$, and 0.15 , respectively) beach sediments. In addition, the positive correlation of $\sum$ REE versus $\mathrm{Fe}_{2} \mathrm{O}_{3}, \mathrm{TiO}_{2}, \mathrm{P}_{2} \mathrm{O}_{5}, \mathrm{Cr}, \mathrm{Ni}, \mathrm{V}, \mathrm{Zn}$, and $\mathrm{Y}$ is significant for the Riachuelos sediments $(r=$ $0.86,0.70,0.53,0.83,0.77,0.80,0.81$, and 0.67 , respectively), which indicates the association of REE with accessory heavy minerals. In contrast, the variations in correlation coefficient observed in the Palma Sola beach sediments $(r=0.02,-0.14,0.76,-0.43,0.07,-0.08$, 0.54 , and 0.72 , respectively) suggests that the REE are partially associated with accessory minerals.

\subsubsection{Principal component analysis (PCA)}

The PCA for the Riachuelos beach sediments demonstrates two major factors (Fig. 13; Additional File 1: Supplementary Information 6). Factor F1 reveals significant negative loading, which represents the association of a few major $\left(\mathrm{TiO}_{2}, \mathrm{Fe}_{2} \mathrm{O}_{3}, \mathrm{MnO}\right.$, and $\left.\mathrm{MgO}\right)$, trace $(\mathrm{V}, \mathrm{Y}$, and $\mathrm{Zn}$ ), and rare earth elements (La, Ce, Pr, Nd, Sm,
$\mathrm{Eu}, \mathrm{Gd}, \mathrm{Tb}, \mathrm{Dy}, \mathrm{Ho}, \mathrm{Er}, \mathrm{Tm}, \mathrm{Yb}$, and $\mathrm{Lu}$ ) related to heavy minerals like magnetite and ilmenite. Factor F2 shows positive loading with elements, which is largely associated with detrital materials. On the other hand, PCA for the Palma Sola beach sediments reveals three factors (Additional File 1: Supplementary Information 7). Factor F1 represents high positive loading with the association of alkali ( $\mathrm{K}$ and $\mathrm{Rb}$ ), alkaline $(\mathrm{Ba}, \mathrm{Mg}, \mathrm{Nd}$, and $\mathrm{Ca}$ ), and rare earth elements $(\mathrm{Pr}, \mathrm{Nd}, \mathrm{Sm}, \mathrm{Gd}$, $\mathrm{Tb}, \mathrm{Dy}, \mathrm{Ho}, \mathrm{Er}, \mathrm{Tm}, \mathrm{Pb}$, and $\mathrm{Lu}$ ), related to the detrital origin. Factor F2 shows a negative loading for elements of $\mathrm{Be}, \mathrm{Cu}, \mathrm{Ga}, \mathrm{Li}, \mathrm{Sn}, \mathrm{Ta}$, Th, and $\mathrm{Zn}$. Factor F3 represents a negative loading with variables of $\mathrm{TiO}_{2}, \mathrm{Fe}_{2} \mathrm{O}_{3}, \mathrm{Co}, \mathrm{Cr}, \mathrm{Hf}, \mathrm{V}$, and $\mathrm{Zr}$, which are associated with heavy minerals like ilmenite, magnetite, chromite, and zircon. Therefore, the combined results obtained from the Pearson's Correlation Coefficient and the Principal Component Analysis indicate the association of major and trace elements with detrital materials rather than biogenic components. 

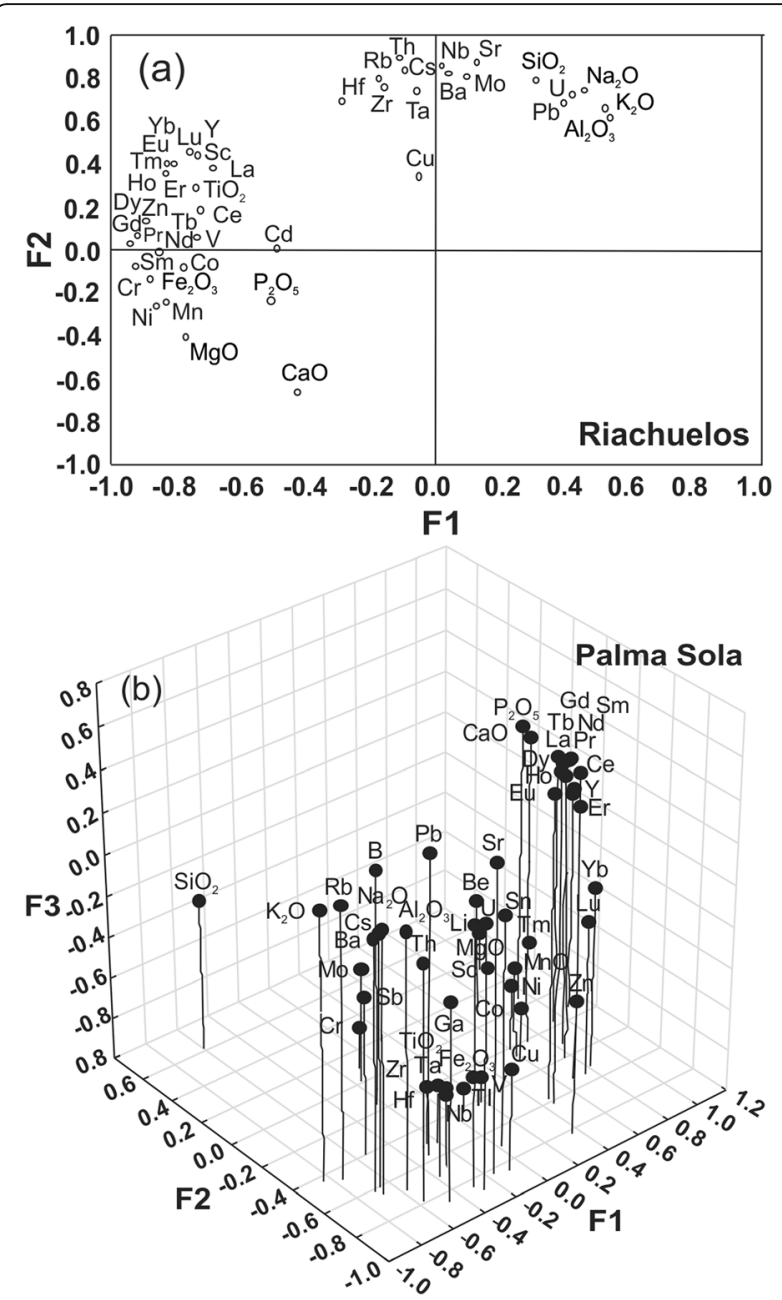

Fig. 13 Bivariate plots of factor F1-factor F2-factor F3 for the bulk geochemistry data of the $\mathbf{a}$ Riachuelos and $\mathbf{b}$ Palma Sola beach sediments

\subsection{Chemical weathering}

Numerous weathering indices like Chemical Index of Alteration $\quad\left(\mathrm{CIA}=100 \times\left[\mathrm{Al}_{2} \mathrm{O}_{3} /\left(\mathrm{Al}_{2} \mathrm{O}_{3}+\mathrm{CaO}+\mathrm{Na}_{2} \mathrm{O}+\mathrm{K}_{2} \mathrm{O}\right)\right]\right.$; Nesbitt and Young 1982) and Chemical Index of Weathering $\left(\mathrm{CIW}=100 \times\left[\mathrm{Al}_{2} \mathrm{O}_{3} /\left(\mathrm{Al}_{2} \mathrm{O}_{3}+\mathrm{CaO}+\mathrm{Na}_{2} \mathrm{O}\right)\right] ;\right.$ Harnois 1988) have been widely used to infer intensity of weathering (e.g., Ota et al. 2017; Anaya-Gregorio et al. 2018; Raza and Mondal 2018; Tawfik et al. 2018; Hossain et al. 2018; Kettanah et al. 2020). However, a few authors (Cullers 2000; Buggle et al. 2011; Garzanti et al. 2015) documented that sediments are affected by various factors like sorting, recycling, and having significant proportions of carbonate or phosphate minerals. Hence, care should be taken when applying CIA and $\mathrm{CIW}$ indices to infer the intensity of weathering, because $\mathrm{CaO}$ content in sediments decrease the values and may mislead interpretation. These authors further recommended to eliminate the $\mathrm{CaO}$ content from the CIA and CIW indices and suggested a simply modified index called CIX (CIX = $\left.100 \times\left[\mathrm{Al}_{2} \mathrm{O}_{3} /\left(\mathrm{Al}_{2} \mathrm{O}_{3}+\mathrm{Na}_{2} \mathrm{O}+\mathrm{K}_{2} \mathrm{O}\right)\right]\right)$, which eliminated
$\mathrm{CaO}$ content (Cullers 2000; Buggle et al. 2011; Garzanti et al. 2014). In general, similar to other indices, the CIX values increase if weathering increases and in highly weathered sediments the values are always more than 80 . The calculated CIX values for the Riachuelos and Palma Sola beach sediments vary from 74 to 77 and from 71 to 74 , respectively (Table 2), suggesting a moderate intensity of chemical weathering for the source area.

In clastic sediments, $\mathrm{Th} / \mathrm{U}, \mathrm{Rb} / \mathrm{Sr}$, and $\mathrm{Ba} / \mathrm{Sr}$ ratios can be used as indicators to infer intensity of weathering, because $\mathrm{Th}, \mathrm{U}, \mathrm{Rb}, \mathrm{Ba}$, and $\mathrm{Sr}$ are sensitive to chemical weathering and their values are always high in extremely weathered sediments (McLennan et al. 1993; Xu et al. 2010). Similarly, Th/U ratios in sediments increase with increasing weathering due to oxidation and loss of uranium. Th/U ratios above four are expected to be indicative of weathering (McLennan et al. 1993). The average $\mathrm{Rb} / \mathrm{Sr}$ and $\mathrm{Ba} / \mathrm{Sr}$ ratios in the Riachuelos $(0.09 \pm 0.01$ and $1.17 \pm 0.08$, respectively) and Palma Sola (0.18 \pm 0.02 and $1.79 \pm 0.18$, respectively) beach sediments are lower than in UCC (0.32 and 1.57, respectively; Taylor and McLennan 1985). Similarly, average Th/U ratio in the Riachuelos and Palma Sola beach sediments are both less than 4 (3.0 \pm 0.7 and $3.3 \pm 0.3$, respectively; Table 3$)$. Hence, the $\mathrm{Rb} / \mathrm{Sr}, \mathrm{Ba} / \mathrm{Sr}$, and $\mathrm{Th} / \mathrm{U}$ ratios in the Riachuelos and Palma Sola beach sediments suggest that the intensity of weathering is moderate in the source area.

\subsection{Source rock composition and tectonic setting}

Trace element concentrations (e.g., Th, U, Y, Sc, Cr, Co, $\mathrm{Ni}, \mathrm{V}$, and REE) in clastic sediments are more reliable indicators of provenance than major elements and are extensively applied in various studies to infer sediment provenance (e.g., Basu 2017; Zhang et al. 2018; Casse et al. 2019). This is because incompatible trace elements are concentrated in sediments derived from felsic igneous rocks, whereas compatible elements are enriched in sediments derived from mafic igneous rocks and their weathered products. Then, elemental ratios such as La/ $\mathrm{Sc}, \mathrm{Th} / \mathrm{Co}$, and $\mathrm{Th} / \mathrm{Sc}$ are significantly different between felsic and mafic rocks and can be used to infer sediment provenance (Cullers and Podkovyrov 2002). Bivariate (Fig. 14a, b) and ternary diagrams (Fig. 14c, d) show that the Riachuelos and Palma Sola beach sediments are plotted between dacite and andesite average compositions, suggesting the provenance of felsic and intermediate igneous rocks. Less contributions from mafic rocks are also revealed by the lower concentrations of $\mathrm{Co}, \mathrm{Ni}$, and $\mathrm{V}$ in the Riachuelos and Palma Sola beach sediments relative to in the UCC (Fig. 8b).

The Riachuelos and Palma Sola beach sediments are little fractionated (LREE/HREE $=\sim 7.16-9.54$ and $\sim 6.23-8.56$, respectively) and are characterized by both negative and 

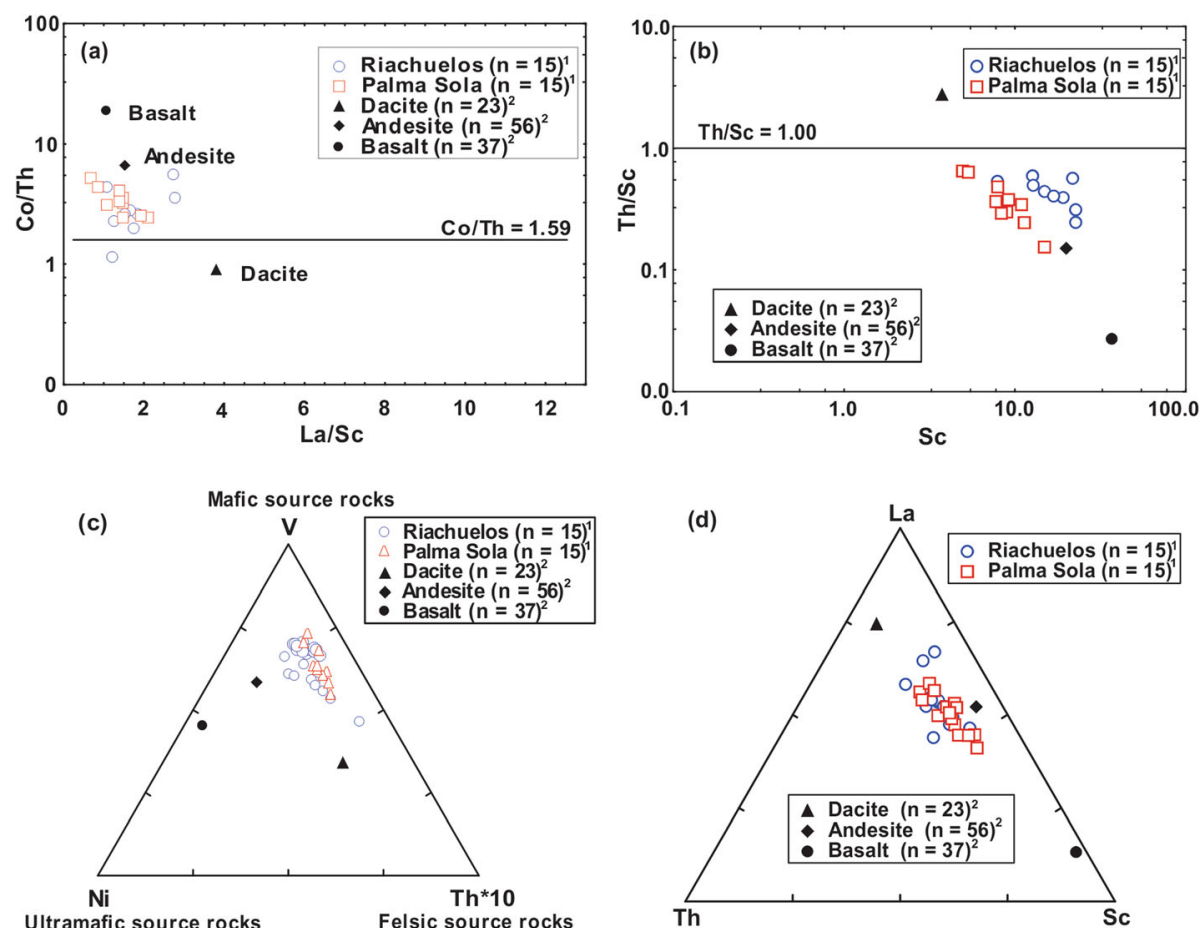

Fig. 14 Bivariate and ternary plots for the Riachuelos and Palma Sola beach sediments: a La/Sc versus Co/Th; b Sc versus Th/Sc; c Ni-Th ${ }^{*} 10-V ; \mathbf{d}$ Th-Sc-La. ${ }^{1}$ This study; ${ }^{2}$ The average compositions of dacite, andesite, and basalt plotted in these diagrams are compiled from Verma (2015), Verma et al. (2016), and Torres-Sánchez et al. (2019)

positive $\mathrm{Eu}$ anomalies $\left(\mathrm{Eu} / \mathrm{Eu}^{*}=\sim 0.90-1.17\right.$ and $\sim 0.81-$ 1.31, respectively; Table 4), suggesting that the sediments were derived from both felsic and intermediate igneous rocks. Furthermore, the chondrite-normalized REE patterns of the Riachuelos and Palma Sola beach sediments are compared with potential source rocks such as rhyolite,

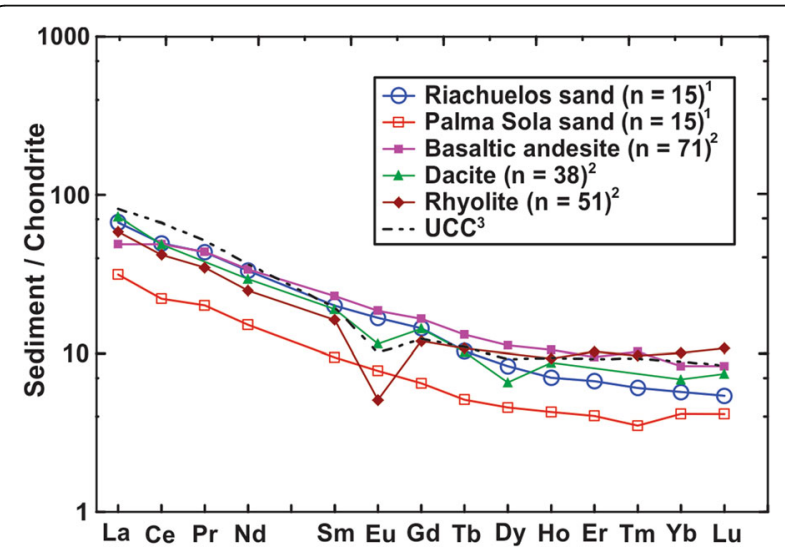

Fig. 15 Chondrite-normalized rare earth element patterns of the Riachuelos and Palma Sola beach sediments compared with the adjacent source rocks in the Gulf of Mexico. ${ }^{1}$ This study; ${ }^{2}$ The average data of basaltic andesite, dacite, and andesite are compiled from Verma (2015), Verma et al. (2016), and Torres-Sánchez et al. (2019); ${ }^{3}$ The Upper Continental Crust (UCC) data are from Taylor and McLennan (1985) dacite, and basaltic andesite located in the Gulf of Mexico coastal areas (Fig. 15). The similarities in REE patterns between the two beach sediments and source rocks are consistent with the interpretation that the detritus was supplied by felsic and intermediate rocks.

The major and trace element concentrations of detrital sediments have been widely used in various studies to infer the tectonic setting of a sedimentary basin, because detrital sediment composition varies significantly between active and passive margin settings (Bhatia 1983; Roser and Korsch 1986; Saha et al. 2010, 2018; Verma and Armstrong-Altrin 2013; Armstrong-Altrin 2015). Although many studies continuously use the old tectonic discrimination diagrams of Bhatia (1983) and Roser and Korsch (1986), in this study, the recently proposed discriminant function diagrams of Verma and ArmstrongAltrin (2016) are considered (Fig. 16). These new statistically discriminant diagrams are efficient to discriminate siliciclastic sediments of island or continental arc, continental rift, and/or collision settings. On these diagrams, the Riachuelos (Fig. 16a, c) and Palma Sola (Fig. 16b, d) beach sediments are divided well within the rift and passive margin fields, suggesting a passive margin setting for the beach areas, which is consistent with the tectonic setting of the Gulf of Mexico (Verma et al. 2016; Centeno-García 2017). 


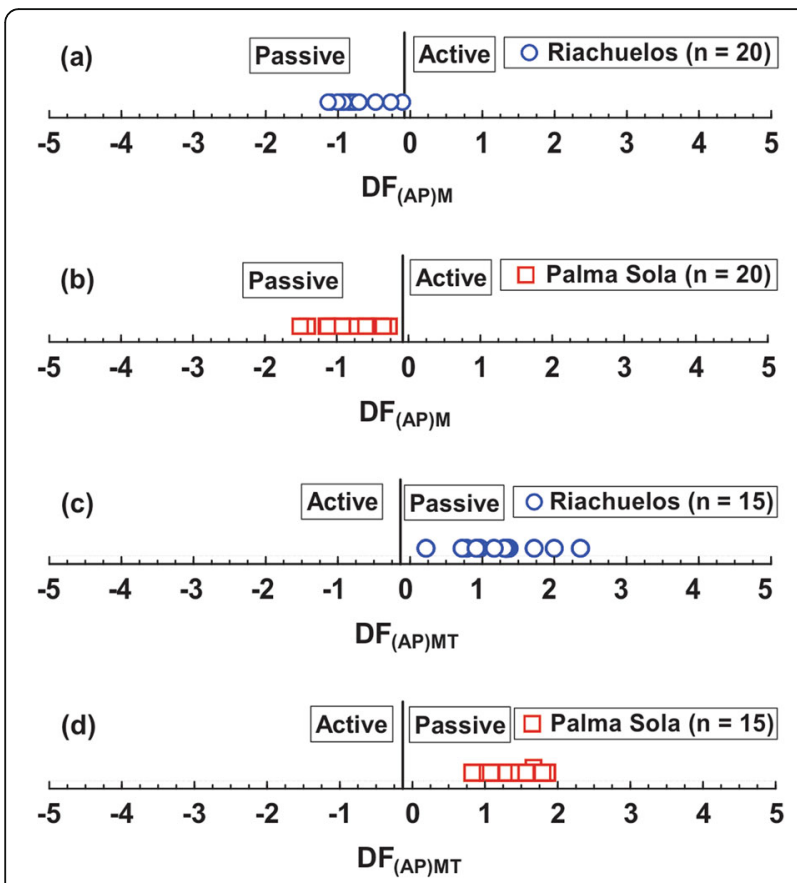

Fig. 16 Tectonic discrimination diagrams for the Riachuelos (blue circles) and Palma Sola (red squares) beach sediments, based on a and $\mathbf{b}$ major element and $\mathbf{c}$ and $\mathbf{d}$ trace element concentrations (after Verma and Armstrong-Altrin 2016). The function DF(AP)M is derived from an equation: $\mathbf{D F}_{(\mathbf{A P}) \mathbf{M}}=\left(3.0005 \times \mathrm{ilr} 1_{\mathrm{TiM}}\right)+(-2.8243 \times$ ilr2 $\left.2_{\text {AIM }}\right)+\left(-1.0596 \times\right.$ ilr $\left._{\text {FeM }}\right)+\left(-0.7056 \times\right.$ ilr4 $\left._{\text {MnM }}\right)+(-0.3044 \times$ ilr5 $\left.5_{\mathrm{MgM}}\right)+\left(0.6277 \times\right.$ ilr6 $\left._{\mathrm{CaM}}\right)+\left(-1.1838 \times\right.$ ilr $\left._{\mathrm{NaM}}\right)+(1.5915 \times$ ilr8 $\left.\mathrm{KM}_{\mathrm{MM}}\right)+\left(0.1526 \times\right.$ ilr $\left._{\mathrm{PM}}\right)-5.9948$. The function $\mathrm{DF}_{\text {(AP)MT }}$ is derived from an equation: $\quad \mathbf{D F}_{(\mathrm{AP}) \mathrm{MT}}=\left(3.2683 \times \mathrm{ilr}_{\mathrm{TiMT}}\right)+\left(5.3873 \times \mathrm{ilr}_{\mathrm{AIMT}}\right)+(1.5546 \times$ ilr3 $\left._{\text {FeMT }}\right)+\left(3.2166 \times\right.$ ilr4 $\left._{\text {MnMT }}\right)+\left(4.7542 \times\right.$ ilr $\left._{\text {MgMT }}\right)+(2.0390 \times$

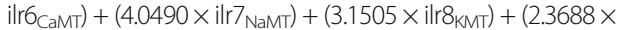
ilr9 PMT $)+\left(2.8354 \times\right.$ ilr10 $\left.0_{\text {CrMT }}\right)+\left(0.9011 \times\right.$ ilr1 $\left._{\text {NbMT }}\right)+(1.9128 \times$ ilr12 $\left.{ }_{\text {NiMT }}\right)+\left(2.9094 \times\right.$ ilr13 $\left.{ }_{V M T}\right)+\left(4.1507 \times\right.$ ilr14 $\left.4_{\text {YMT }}\right)+(3.4871 \times$ il $\left.15_{\mathrm{ZMMT}}\right)-3.2088$

\subsection{Probable provenance based on zircon surface microtextures}

The microtextures identified on zircon grain surfaces (Figs. 6, 7) and their implications on provenance are briefly discussed.

The collision fractures, V-shaped percussion cracks, meandering ridges, arc and linear steps indicate highenergy grain to grain impact and mechanical grinding by littoral transport, as well as high-energy subaqueous deposition such as in river and coastal environments (Finzel 2017; Hossain et al. 2020). However, the mechanical features on the grain surface may likely be associated with the hydrodynamic conditions during littoral transport and wave action in the nearshore zone (KalińškaNartiša et al. 2018). The abraded grains with bulbous edges identified in a few zircons show the evidence of high-energy collision through saltation in an aeolian environment (Mahaney 2002; Chmielowska and Woronko 2019). Angular grains with broken edges indicate a short transport in littoral environment as well as a highenergy subaqueous environment, where the breaking of waves is probably the transport agent (Costa et al. 2017). Conchoidal fracture is one of the most dominant microtextures on zircon surfaces identified in both beach areas, which indicate liberation of grains from crystalline rocks (Madhavaraju et al. 2009). In addition, collisions between zircon grains and pebbles may also cause fracturing, as well as delamination (Vos et al. 2014).

The etching process is revealed by chemical features like solution pits and precipitation features associated with the percolation of seawater. The silica globules, silica pellicles, and silica flowers indicate that zircon grains were subjected to precipitation with silica saturated solutions for a considerable time. Vos et al. (2014) documented that when precipitation on grain surface continues, silica globules start to merge to form silica flowers and pellicles.

Overall, the mechanical and chemical features identified on the zircon surfaces of the Riachuelos and Palma Sola beach sediments suggest their transport processes by both littoral and aeolian currents, and a high-energy subaqueous coastal depositional environment.

\subsection{Probable provenance based on detrital zircon $\mathrm{U}-\mathrm{Pb}$ ages} $\mathrm{U}-\mathrm{Pb}$ dating ages of the detrital zircons of the Riachuelos and Palma Sola beach sediments are compared with other zircon $\mathrm{U}-\mathrm{Pb}$ ages reported by various authors from different terranes of Gulf of Mexico (Fig. 17), and are briefly discussed, to infer the provenance.

\subsubsection{Relative zircon age probability distribution - Proterozoic}

The Riachuelos beach sediments own three Proterozoic zircons with $\mathrm{U}-\mathrm{Pb}$ dating ages varying from $1370 \mathrm{Ma}$ to 903.8 Ma and the Palma Sola beach sediments own nine Proterozoic zircons with $\mathrm{U}-\mathrm{Pb}$ ages varying from 1652 $\mathrm{Ma}$ to $629.9 \mathrm{Ma}$. The possible source terranes contributed to Proterozoic zircons of the Riachuelos and Palma Sola beach areas are the Chiapas Massif Complex and the Oaxaca Complex, where the documented zircon $\mathrm{U}-\mathrm{Pb}$ ages vary from $\sim 1500 \mathrm{Ma}$ to $\sim 450 \mathrm{Ma}$ (Keppie et al. 2003; Weber et al. 2012). In addition, Escalona-Alcázar et al. (2016) and Wengler et al. (2019) also reported Proterozoic zircons in the Mesa Central Province, and they inferred that Oaxaca Complex and Chiapas Massif Complex are potential source terranes for the Mesa Central Province.

\subsubsection{Relative zircon age probability distribution - Paleozoic} Detrital zircons of the Riachuelos and Palma Sola beach areas also show the Paleozoic ages $(\sim 480-252.5 \mathrm{Ma})$. However, Riachuelos beach sediments are more dominated by the Paleozoic zircons $(n=30)$ than Palma Sola beach sediments $(n=15)$. The most likely potential source terrane for the Paleozoic zircons of beach areas, 

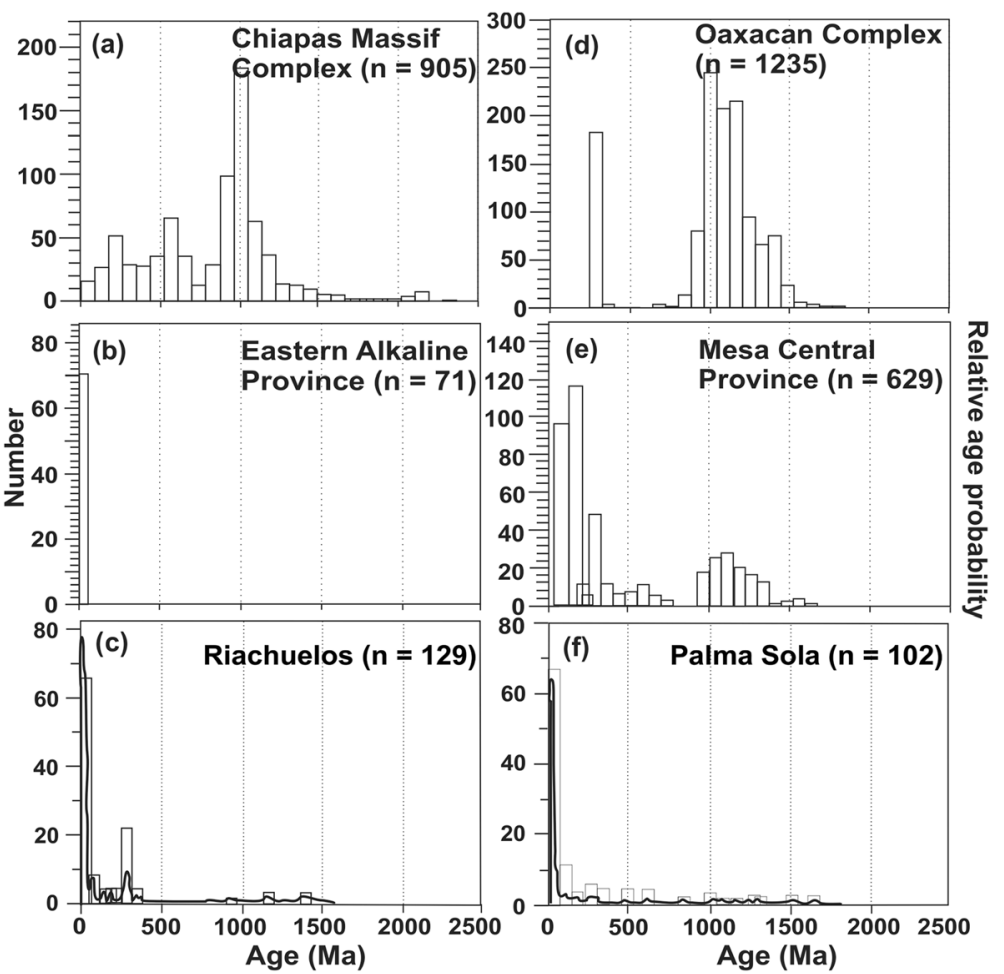

Fig. 17 Relative probability distribution of detrital zircon U-Pb ages of the Riachuelos and Palma Sola beach sediments in this study and from four terranes in the SW Gulf of Mexico (referring Figs. 1 and 2 for locations). a Chiapas Massif Complex (Weber et al. 2009, 2018; Cisneros de León et al. 2017); b Eastern Alkaline Province (Cantagrel and Robin 1979; López-Infanzón 1991; Nelson and González-Caver 1992; Ferrari et al. 2005; Solari et al. 2011); c Riachuelos beach area (this study); d Oaxacan Complex (Ortega-Obregón et al. 2014); e Mesa Central Province (EscalonaAlcázar et al. 2016; Wengler et al. 2019); f Palma Sola beach area (this study). $n=$ Numbers of zircons

is the Mesa Central Province, located near to Zacatecas City, Mexico. The Mesa Central Province consists of Zacatecas, Nazas, La Boca, and La Joya sedimentary formations (Barboza-Gudiño et al. 2010). Wengler et al. (2019) reported that the zircon populations from the Zacatecas and Naza Formations vary between 350$215 \mathrm{Ma}$ and $\sim 440-200 \mathrm{Ma}$, respectively. These ages are similar to the zircon ages inferred from the Riachuelos and Palma Sola beach areas. Then it can be assumed that the Mesa Central Province is also one of the source terranes, which supplied sediments to the beach areas. The Zacatecas and Nazas Formations in the Mesa Central Province are composed of marine-continental siliciclastic sediments with thin-bedded turbidites, which consist of sandstone, shale and conglomerates (AguillónRobles et al. 2014; Centeno-García 2017; RamosVázquez and Armstrong-Altrin 2019; Torres-Sánchez et al. 2019; Verma et al. 2019).

\subsubsection{Relative zircon age probability distribution - Mesozoic} Mesozoic zircons are identified in the Riachuelos ( 19466.1 Ma, $n=9)$ and Palma Sola $(\sim 185.5-72.4 \mathrm{Ma}, n=9)$ beach sediments. The probable source of Mesozoic zircons in Riachuelos and Palma Sola beach areas is the Chiapas Massif Complex, because similar Mesozoic zircon ages from the Chiapas Massif Complex are also reported by previous studies (e.g., Weber et al. 2009; Cisneros de León et al. 2017).

5.5.4 Relative zircon age probability distribution - Cenozoic Detrital zircon $\mathrm{U}-\mathrm{Pb}$ dating results (Additional File 1: Supplementary Information 4 and 5) reveal that the Riachuelos and Palma Sola beach sediments are dominated by the Cenozoic zircons $(\sim 33.1-0.39 \mathrm{Ma}$ and $\sim 64.9-0.4$ Ma, respectively; Figs. 12, 17). The potential source for these Cenozoic zircons is the Eastern Alkaline Province, located along the coastal region of the SW Gulf of Mexico and formed along the Gulf-parallel extensional fault (Robin and Tournon 1978; Robin 1982). The Eastern Alkaline Province consists of alkaline volcanics dominated by basaltic andesites. The detrital zircon ages reported for the Eastern Alkaline Province vary from 65 Ma to $0.18 \mathrm{Ma}$ (Cantagrel and Robin 1979; López-Infanzón 1991; Nelson and González-Caver 1992; Ferrari et al. 2005). Thus the Eastern Alkaline Province is supposed as the provenance for the Cenozoic zircons of the Riachuelos and Palma Sola beach sediments. 


\section{Conclusions}

Zircon, apatite, magnetite, titano-magnetite, and ilmenite were identified in the Riachuelos and Palma Sola beach sediments. The zircon grain morphology and microtextures revealed that sediments were transported by littoral and aeolian currents and deposited in a highenergy subaqueous environment. In comparison with the Palma Sola beach sediments, Riachuelos beach sediments were lower in $\mathrm{SiO}_{2}$ and higher in Sc, V, Co, and $\mathrm{Ni}$ contents, suggesting a higher proportion of detritus derived from intermediate igneous rocks. Statistical analysis revealed that the major, trace and rare earth elements were generally associated with terrigenous rather than biogenic materials. The chondrite-normalized REE patterns for the Riachuelos and Palma Sola beach sediments were enriched in LREE, with negative and positive $\mathrm{Eu}$ anomalies, indicating their felsic and intermediate igneous sources. A passive margin setting inferred from the tectonic discriminant function diagrams for the beach areas was consistent with the geological setting of the Gulf of Mexico.

$\mathrm{Th} / \mathrm{U}$ ratios of zircon grains were $>0.1$, indicating a magmatic origin for the Riachuelos and Palma Sola beach sediments. The Riachuelos and Palma Sola beach sediments were dominated by the Paleozoic and Cenozoic zircons, and Proterozoic zircons were limited. The Zacatecas and Nazas Formations in the Mesa Central Province were the most likely source for Paleozoic detrital zircons. The Cenozoic detrital zircons implied that the source terrane, which delivered sediments to the Riachuelos and Palma Sola beach areas, was the nearby Eastern Alkaline Province. Significant discrimination in zircon REE patterns with respect to age was not identified, which suggested that the zircon $\mathrm{U}-\mathrm{Pb}$ geochronology is a better method to identify sediment provenance than zircon REE patterns and abundances. The resemblance in zircon surface microtextures and zircon age clusters between the Riachuelos and Palma Sola beach sediments demonstrated a similarity in their source rocks, transport distance from the source area, and wave energy.

\section{Supplementary information}

Supplementary information accompanies this paper at https://doi.org/10. 1186/s42501-020-00075-9.

\footnotetext{
Additional file 1 Supplementary Information 1: Quantitative compositional data of sand grains from the Riachuelos and Palma Sola beach sediments obtained by Scanning Electron Microscopy (SEM) with Energy Dispersive X-Ray Spectroscopy (EDS). Supplementary Information 2: Trace ( $\mathrm{Nb}, \mathrm{Hf}, \mathrm{Th}$, and $\mathrm{U}$ ) and rare earth element data (in ppm) for the detrital zircon grains in the Riachuelos (Sample No. RIA4) beach sediments, Gulf of Mexico. Supplementary Information 3: Trace (Nb, $\mathrm{Hf}$, Th, and $\mathrm{U}$ ) and rare earth element data (in ppm) for the detrital zircon grains in the Palma Sola (Sample No. PAL20) beach sediments, Gulf of Mexico. Supplementary Information 4: $\mathrm{U}-\mathrm{Pb}$ ages of detrital zircons collected from the Riachuelos (Sample No. RIA4) beach sediments, Gulf of
}

Mexico. Supplementary Information 5: U-Pb ages of detrital zircons collected from the Palma Sola (Sample No. PAL20) beach sediments, Gulf of Mexico. Supplementary Information 6: Factor loadings for the Riachuelos beach sediments, Gulf of Mexico. Supplementary Information 7: Factor loadings for the Palma Sola beach sediments, Gulf of Mexico.

Additional file 2 Supplementary Information 8: Post-ablation cathodoluminescence images of representative detrital zircons from the Riachuelos beach sediments. Supplementary Information 9: Post-ablation cathodoluminescence images of representative detrital zircons from the Palma Sola beach sediments.

\section{Acknowledgements}

Financial assistance extended by the CONACyT Ciencia Basica (A1-S-21287) and PAPIIT (IN107020) projects are highly acknowledged. I appreciate Mario Marca Castillo for sample collection along the Riachuelos and Palma Sola beach areas. I gratefully acknowledge Mayla A. Ramos-Vázquez and Vanessa del Carmen for their help in zircon separation from beach sediments. I am indebted to Susana Santiago-Perez, Edwardo Alfredo Morales, and Ricardo Martínez Domínguez for their assistance in the Sedimentology Laboratory during sample preparation. I thank the staffs Patricia Girón García, Carlos LinaresLópez, Laura Elena Gómez Lizárraga, and Teodoro Hernández Treviño for their help in XRD, SEM-EDS, SEM, and mineral identification. I also like to thank Dr. Luigi Solari, Carlos Ortega-Obregón, and Harim Arvizu Gutiérrez at the Laboratorio de Estudios Isotópicos, for their assistance in zircon $\mathrm{U}-\mathrm{Pb}$ dating by LA-ICP-MS. I appreciate Edith Xiadani C. Zárate, "Ayudante de Investigador Nacional Nivel III", for the help in the preparation of Supplementary Tables. I express my sincere gratefulness to the editor and three anonymous reviewers for their excellent suggestions, which significantly improved the presentation.

\section{Author's contributions}

AAJS wrote the manuscript and approved the final revised version.

\section{Funding}

This study was supported by the 1) Consejo Nacional de Ciencia y Tecnología (CONACyT) (Project No. A1-S-21287); 2) Dirección General de Asuntos del Personal Académico (DGAPA) — Programa de Apoyo a Proyectos de Investigación e Innovación Tecnología (PAPIIT) (Project No. IN107020); and, 3) Instituto de Ciencias del Mar y Limnologia Institutional Fund (Project No. 616).

\section{Availability of data and materials}

All data discussed in this study are available in the current Tables, Figures, and Supplementary material (Additional Files 1 and 2: Supplementary Information 1-9) of this manuscript.

\section{Competing interests}

The author declares that he has no competing interests; and, there are no other persons who satisfied the criteria for authorship but are not listed.

Received: 16 April 2020 Accepted: 16 September 2020

Published online: 09 October 2020

\section{References}

Aguillón-Robles, A., M. Tristán-González, G. Jesús Aguirre-Díaz, R.A. LópezDoncel, H. Bellon, and G. Martínez-Espara. 2014. Eocene to quaternary mafic-intermediate volcanism in San Luis Potosí, Central Mexico: The transition from Farallon plate subduction to intra-plate continental magmatism. Journal of Volcanology and Geothermal Research 276: 152172.

An, W., H.-W. Kuang, Y.-Q. Liu, N. Peng, K.-M. Xu, H. Xu, P. Zhang, K.-B. Wang, S.-Q. Chen, and Y.-X. Zhang. 2016. Detrital zircon dating and tracing the provenance of dinosaur bone beds from the late cretaceous Wangshi Group in Zhucheng, Shandong, East China. Journal of Palaeogeography 5 (1): 72-99.

Anaya-Gregorio, A., J.S. Armstrong-Altrin, M.L. Machain-Castillo, P.C. MontielGarcía, and M.A. Ramos-Vázquez. 2018. Textural and geochemical characteristics of late Pleistocene to Holocene fine-grained deep-sea 
sediment cores (GM6 and GM7), recovered from southwestern Gulf of Mexico. Journal of Palaeogeography 7 (3): 253-271.

Armstrong-Altrin, J.S. 2015. Evaluation of two multi-dimensional discrimination diagrams from beach and deep sea sediments from the Gulf of Mexico and their 6application to Precambrian clastic sedimentary rocks. International Geology Review 57: 1446-1461.

Armstrong-Altrin, J.S., and O. Natalhy-Pineda. 2014. Microtextures of detrital sand grains from the Tecolutla, Nautla, and Veracruz beaches, western Gulf of Mexico, Mexico: Implications for depositional environment and palaeoclimate. Arabian Journal of Geosciences 7: 4321-4333.

Bansal, U., S. Banerjee, D.K. Ruidas, and K. Pande. 2018. Origin and geochemical characterization of the glauconites in the upper cretaceous Lameta formation, Narmada Basin, Central India. Journal of Palaeogeography 7 (2): 99-116.

Barboza-Gudiño, J.R., A. Zavala-Monsiváis, G. Venegas-Rodríguez, and L.D. Barajas-Nigoche. 2010. Late Triassic stratigraphy and facies from northeastern Mexico: Tectonic setting and provenance. Geosphere 6: 621-640.

Barros dos Santos, J.C., E. Le Pera, C. Souza de Oliveira, V. Severino de Souza Júnior, P. Fabrício de Araújo, M.M. Corrêa, and A. Carlos de Azevedo. 2019. Impact of weathering on REE distribution in soil-saprolite profiles developed on orthogneisses in Borborema Province, NE Brazil. Geoderma 347: 103-117.

Basu, A. 2017. Evolution of siliciclastic provenance inquiries: A critical appraisal. In Sediment Provenance. Chapter 2, ed. Rajat Mazumder, 5-23. Netherlands: Elsevier Amsterdam.

Basu, A., M.E. Bickford, and R. Deasy. 2016. Inferring tectonic provenance of siliciclastic rocks from the chemical compositions: A dissent. Sedimentary Geology 336: 26-35.

Bhatia, M.R. 1983. Plate tectonics and geochemical composition of sandstones. Journal of Geology 91: 611-627.

Bhattacharjee, J., K.K. Ghosh, and B. Bhattacharya. 2018. Petrography and geochemistry of sandstone-mudstone from Barkar formation (early Permian), Raniganj Basin, India: Implications for provenance, weathering and marine depositional conditions during Gondwana sedimentation. Geological Journal 53: 1102-1122.

Boyd, R., R. Dalrymple, and B.A. Zaitlin. 1992. Classification of clastic coastal depositional environments. Sedimentary Geology 80: 139-150

Buggle, B., B. Glaser, U. Hambach, N. Gerasimenko, and S. Markovic. 2011. An evaluation of geochemical weathering indices in loess-paleosol studies. Quaternary International 240: 12-21.

Cantagrel, J.M., and C. Robin. 1979. K-Ar dating on eastern Mexican volcanic rocks-relations between the andesitic and the alkaline provinces. Journal of Volcanology and Geothermal Research 5: 99-114.

Carranza-Edwards, A., J.J. Kasper-Zubillaga, R.G. Martínez-Serrano, M. CabreraRamírez, L. Rosales-Hoz, M.A.A. Mendieta, A.Z. Márquez-García, and R.L. Santa-Cruz. 2019. Provenance inferred through modern beach sands from the Gulf of Tehuantepec, Mexico. Geological Journal 54: 552-563.

Carranza-Edwards, E. 2011. Mexican littoral of the Gulf of Mexico. In Gulf of Mexico: Origin, Waters and Biota, Volume 3, Geology, ed. N.A. Buster and C.W. Holmes, 293-296. College Station: Texas A\&M University Press.

Casse, M., J.-C. Montero-Serrano, G. St-Onge, and A. Poirier. 2019. REE distribution and $\mathrm{Nd}$ isotope composition of estuarine waters and bulk sediment leachates tracing lithogenic inputs in eastern Canada. Marine Chemistry 211: 117-130.

Centeno-García, E. 2017. Mesozoic tectono-magmatic evolution of Mexico: An overview. Ore Geology Reviews 81: 1035-1052.

Chaudhuri, A., K. Das, S. Banerjee, and I.C.W. Fitzsimons. 2020. Detrital zircon and monazite track the source of Mesozoic sediments in Kutch to rocks of late Neoproterozoic and early Palaeozoic orogenies in northern India. Gondwana Research 80: 188-201.

Chmielowska, D., and B. Woronko. 2019. A source of loess-like deposits and their attendant palaeoenvironment - Orava Basin, Western Carpathian Mountains, S. Poland. Aeolian Research 38: 60-76.

Cisneros de León, A., B. Weber, F. Ortega-Gutiérrez, R. González-Guzmán, R. Maldonado, L. Solari, P. Schaaf, and R. Manjarrez-Juárez. 2017. Grenvillian massif-type anorthosite suite in Chiapas, Mexico: Magmatic to polymetamorphic evolution of anorthosites and their Ti-Fe ores Precambrian Research 295: 203-226.

Costa, P.J.M., Y.S. Park, Y.D. Kim, M. Quintela, W.C. Mahaney, F. Dourado, and S. Dawson. 2017. Imprints in silica grains induced during an openchannel flow experiment: Determination of microtextural signatures during aqueous transport. Journal of Sedimentary Research 87: 677-687.

Cullers, R.L. 2000. The geochemistry of shales, siltstones and sandstones of Pennsylvanian-Permian age, Colorado, USA: Implications for provenance and metamorphic studies. Lithos 51: 181-203.

Cullers, R.L., and N.V. Podkovyrov. 2002. The source and origin of terrigenous sedimentary rocks in the Mesoproterozoic Ui group, southeastern Russia. Precambrian Research 117: 157-183.

Davis, R.A. 1988. Morphodynamics of the west-central Florida barrier system: The delicate balance between wave- and tide-domination. In Coastal Lowlands, Geology and Geotechnology, ed. W.J. Van der Linden, S.A. Cloetingh, J.P. Vandenberghe, and W.J. Van De Graaff, 225-235. Dordrecht: Kluwer Academic Publisher.

Escalona-Alcázar, F.J., L. Solari, J.C. García y Barragán, C. Carrillo-Castillo, J. Bluhm-Gutiérrez, P. García-Sandoval, A.F. Nieto-Samaniego, and E.P. Núñez-Peña. 2016. The Palaeocene-early Oligocene Zacatecas conglomerate, Mexico: Sedimentology, detrital zircon U-Pb ages, and sandstone provenance. International Geology Review 58 (7): 826-848.

Etemad-Saeed, N., M. Hosseini-Barzi, M.H. Adabi, A. Sadeghi, and A. Houshmandzadeh. 2015. Provenance of Neoproterozoic sedimentary basement of northern Iran, Kahar formation. Journal of African Earth Sciences 111: 54-75.

Ferrari, L., T. Tagami, M. Equchi, M.T. Orozco-Esquivel, C.M. Petrone, J. Jacobo-Albarrán, and M. López-Martínez. 2005. Geology, geochronology and tectonic setting of late Cenozoic volcanism along the southwestern Gulf of Mexico: The eastern Alkaline Province revisited. Journal of Volcanology and Geothermal Research 146: 284-306.

Finzel, E.S. 2017. Detrital zircon microtextures and U-Pb geochronology of upper Jurassic to Paleocene strata in the distal north American cordillera foreland basin. Tectonics 36: 1295-1316.

Garzanti, E., M. Pardon, M. Setti, A. López-Galindo, and I.M. Villa. 2014 Provenance versus weathering on the composition of tropical river mud (southern Africa). Chemical Geology 366: 61-74.

Garzanti, E., A. Resentini, S. Andó, G. Vezzoli, A. Pereira, and P. Vermeesch. 2015. Physical controls on sand composition and relative durability of detrital minerals during ultra-long distance littoral and aeolian transport (Namibia and southern Angola). Sedimentology 62: 971-996.

Greggio, N., B.M.S. Giambastiani, B. Campo, and E. Dinelli. 2018. Sediment composition, provenance, and Holocene paleoenvironmental evolution of the southern Po River coastal plain (Italy). Geological Journal 53: 914-928.

Harnois, L. 1988. The CIW index: A new chemical index of weathering Sedimentary Geology 55 (3-4): 319-322.

Hernández-Hinojosa, V., P.C. Montiel-García, J.S. Armstrong-Altrin, R. Nagarajan, and J.J. Kasper-Zubillaga. 2018. Textural and geochemical characteristics of beach sands along the western Gulf of Mexico, Mexico. Carpathian Journal of Earth and Environmental Science 13: 161174

Hossain, H.M., Q.H. Hossain, A. Kamei, and D. Araoka. 2018. Compositional variations, chemical weathering, and provenance of sands from Cox's bazar and Kuakata beach areas, Bangladesh. Arabian Journal of Geosciences 11: 749.

Hossain, H.M.Z., J.S. Armstrong-Altrin, A.H.M.N. Jamil, M.M. Rahman, C.J. Hernández-Coronado, and M.A. Ramos-Vázquez. 2020. Microtextures on quartz grains in the Kuakata beach, Bangladesh: Implications for provenance and depositional environment. Arabian Journal of Geosciences 13: 291.

Inman, D.L., and C.E. Nordstrom. 1971. On the tectonic and morphologic classification of coasts. The Journal of Geology 79 (1): 1-21.

Jarvis, K.E. 1988. Inductively coupled plasma mass spectrometry: A new technique for the rapid or ultra-level determination of the rare-earth elements in geological materials. Chemical Geology 68: 31-39.

Ji, H., H. Tao, Q. Wang, D. Ma, and L. Hao. 2019. Petrography, geochemistry, and geochronology of lower Jurassic sedimentary rocks from the 
northern Tianshan (Wesr Bogda area), Northwest China: Implications for provenance and tectonic evolution. Geological Journal 54: 1688-1714.

Joy, S., S. Patranabis-Deb, D. Saha, H. Jelsma, R. Maas, U. Söderlund, S. Tappe, G. van der Linde, and A. Banerjee. 2019. Depositional history and provenance of cratonic "Purana" basins in southern India: A multipronged geochronology approach to the Proterozoic Kaladgi and Bhima basins. Geological Journal 54 (5): 2957-2979.

Kalińška-Nartiša, E., N. Stivrins, and I. Grudzinska. 2018. Quartz grains reveal sedimentary palaeoenvironment and past storm events: A case study from eastern Baltic. Estuarine Coastal and Shelf Sciences 200: 359-370.

Karudu, T.K. 2019. Heavy mineral distribution and provenance studies of coastal sediments of Visakhapatnam coast: Statistical approach. Journal of the Indian Association of Sedimentologists 36 (2): 64-85.

Kasper-Zubillaga, J.J., E. Arellano-Torres, and J.S. Armstrong-Altrin. 2019. Physical degradation and early diagenesis in foraminiferal tests after subaerial exposure in terrigenous-depleted beaches of Yucatan, Mexico. Carbonates and Evaporites 34: 1175-1189.

Keppie, J.D., J. Dostal, K.L. Cameron, L.A. Solari, F. Ortega-Gutiérrez, and R. Lopez. 2003. Geochronology and geochemistry of Grenvillian igneous suites in the northern Oaxaca complex, southern Mexico: Tectonic implications. Precambrian Research 120: 365-389.

Kettanah, Y.A., J.S. Armstrong-Altrin, and F.A. Mohammad. 2020. Petrography and geochemistry of siliciclastic rocks of the middle Eocene Gercus formation, northern Iraq: Implications for provenance and tectonic setting. Geological Journal 55. https://doi.org/10.1002/gj.3880.

Li, C., C. Lyu, G. Chen, G. Zhang, M. Ma, H. Yang, and G. Bi. 2019. Zircon U$\mathrm{Pb}$ ages and REE composition constraints on the provenance of the continental slope-parallel submarine fan, western Qiongdongnan Basin, northern margin of the South China Sea. Marine and Petroleum Geology 102: $350-362$

Liu, F., Y. Zhou, Z. Zhang, and B. Yue. 2019. Detrital zircon U-Pb geochronology of early cretaceous sedimentary rocks in Dingzi Bay and Taolin area from the Sulu Orogen: Provenances and tectonic implications. Geological Journal 54: 2693-2715.

López-Infanzón, M. 1991. Petrologic study of the volcanic rocks in the sierra de Chiconquiaco-Palma Sola, Central Veracruz, Mexico. MSc Thesis, Tulane University, New Orleans, USA, 140.

Löwen, K., G. Meinhold, A. Arslan, T. Güngör, and J. Berndt. 2020. Evolution of the Palaeotethys in the eastern Mediterranean: A multi-method approach to unravel the age, provenance and tectonic setting of the upper Palaeozoic Konya complex and its Mesozoic cover sequence (south-Central Turkey). International Geology Review 62 (4): 389-414.

Ludwig, K.R. 2008. ISOPLOT Vers. 4.15. Berkeley Geochronology Center (BGC): http://www.bgc.org/isoplot_etc/isoplot.html.

Madhavaraju, J., J.S. Armstrong-Altrin, R.B. Pillai, and T. Pi-Puig. 2020. Geochemistry of sands from the Huatabampo and Altata beaches. Gulf of California, Mexico. Geological Journal 55: 1-20. https://doi.org/10. 1002/gj.3864.

Madhavaraju, J., J.C.G. Barragán, S.K. Hussain, and S.P. Mohan. 2009. Microtextures on quartz grains in the beach sediments of Puerto Peñasco and Bahia Kino, Gulf of California, Sonora, Mexico. Revista Mexicana de Ciencias Geológicas 26: 367-379.

Madhavaraju, J., J.C. Saucedo-Samaniego, H. Loser, I.G. Espinoza-Maldonado, L. Solari, R. Monreal, F.J. Grijalva-Noriega, and C. Jaques-Ayala. 2018. Detrital zircon record of Mesozoic volcanic arcs in the lower cretaceous mural limestone, northwestern Mexico. Geological Journal 53 (4): 2621-2645.

Mahaney, W.C. 2002. Atlas of sand grain surface textures and applications, 237. New York: Oxford University Press.

Mahaney, W.C., A.G. Fairén, J.M. Dohm, and D.H. Krinsley. 2012. Weathering rinds on clasts: Examples from earth and Mars as short and long term recorders of paleoenvironment. Planetary and Space Science 73: 243253.

Margolis, S.V., and D.H. Krinsley. 1974. Processes of formation and environmental occurrence of microfeatures on detrital quartz grains. American Journal of Science 274: 449-464.

McLennan, S.M., S.R. Hemming, D.K. McDaniel, and G.N. Hanson. 1993. Geochemical approaches to sedimentation, provenance, and tectonics.
Geological Society of America, Special Paper 284: 21-40. https://doi.org/ 10.1130/SPE284-p21.

Men, X., C. Mou, X. Ge, and Y. Wang. 2019. Geochemical characteristics of siliceous rocks of Wufeng formation in the late Ordovician, South China: Assessing provenance, depositional environment, and formation model. Geological Journal 54: 1-21.

Mohammad, A., P.B. Murthy, E.N.D. Rao, and H. Prasad. 2020. A study on textural characteristics, heavy mineral distribution and grainmicrotextures of recent sediment in the coastal area between the Sarada and Gosthani rivers, east coast of India. International Journal of Sediment Research 35 (5): 484-503.

Monreal-Gómez, M.A., and D.A. Salas de León. 1990. Simulación de la circulación de la Bahía de Campeche. Geofísica Internacional 29 (2): 101-111.

Muñoz-Salinas, E., M. Castillo, D. Sanderson, and T. Kinnaird. 2017. Geochronology and landscape evolution of the strand-plain of the Usumacinta and Grijalva rivers, southern Mexico. Journal of South American Earth Sciences 79: 394-400.

Nagarajan, R., J.S. Armstrong-Altrin, F.L. Kessler, E.L. Hidalgo-Moral, D. DodgeWan, and N.I. Taib. 2015. Provenance and tectonic setting of Miocene siliciclastic sediments, Sibuti formation, northwestern Borneo. Arabian Journal of Geosciences 8 (10): 8549-8565.

Nagarajan, R., J.S. Armstrong-Altrin, F.L. Kessler, and J. Jong. 2017. Petrological and geochemical constraints on provenance, paleoweathering and tectonic setting of clastic sediments from the Neogene Lambir and Sibuti formations, northwestern Borneo. In Sediment Provenance. Chapter 7, ed. Rajat Mazumder, 123-153. Netherlands: Elsevier Amsterdam

Ndjigui, P.-D., E.C. Bayiga, V.L. Onana, S. Djenabou-Fadil, and G.S.A. Ngono. 2019. Mineralogy and geochemistry of recent alluvial sediments from the Ngaye River watershed, northern Cameroon: Implications for the surface processes and au-PGE distribution. Journal of African Earth Sciences 150: 136-157.

Nelson, S.A., and E. González-Caver. 1992. Geology and K-Ar dating of the Tuxtla volcanic field, Veracruz. Mexico. Bulletin of Volcanology 55: 85-96.

Nesbitt, H.W., and G.M. Young. 1982. Early Proterozoic climate and plate motions inferred from major element chemistry of lutites. Nature 299 $715-717$.

Ortega-Obregón, C., L.A. Solari, A. Gómez-Tuena, M. Elías-Herrera, F. OrtegaGutiérrez, and C. Macías-Romo. 2014. Permian-carboniferous arc magmatism in southern Mexico: U-Pb dating, trace element and $\mathrm{Hf}$ isotopic evidence on zircons of earliest subduction beneath the western margin of Gondwana. International Journal of Earth Sciences 103 (5): 1287-1300.

Ota, Y., H. Kawahata, T. Sato, and K. Seto. 2017. Flooding history of Lake Nakaumi, western Japan, inferred from sediment records spanning the past 700 years. Journal of Quaternary Science 32 (8): 1063-1074.

Papadopoulos, A., I.T. Tzifas, and H. Tsikos. 2019. The potential for REE and associated critical metals in coastal sand (placer) deposits of Greece: A review. Minerals 9: 469.

Paton, C., J.D. Woodhead, J.C. Hellstrom, J.M. Hergt, A. Greig, and R. Maas. 2010. Improved laser ablation U-Pb zircon geochronology through robust downhole fractionation correction. Geochemistry Geophysics Geosystems 11: Q0AA06. https://doi.org/10.1029/2009GC002618.

Peña-Alonso, T.A., J. Estrada-Carmona, R.S. Molina-Garza, L. Solari, G. Levresse, and C. Latorre. 2017. Lateral spreading of the middle to lower crust inferred from Paleocene migmatites in the Xolapa complex (Puerto Escondido, Mexico): Gravitational collapse of a Laramide orogen? Tectonophysics 706-707: 143-163.

Peña-Alonso, T.A., R.S. Molina-Garza, G. Villalobos-Escobar, J. EstradaCarmona, G. Levresse, and L. Solari. 2018. The opening and closure of the Jurassic-cretaceous Xolapa basin, southern Mexico. Journal of South American Earth Sciences 88: 599-620.

Petrus, J.A., and B.S. Kamber. 2012. VizualAge: A novel approach to laser ablation ICP-MS U-Pb geochronology data reduction. Geostandards Geoanalytical Research 36 (3): 247-270.

Ramírez-Peña, C.F., and G. Chávez-Cabello. 2017. Age and evolution of thinskinned deformation in Zacatecas, Mexico: Sevier orogeny evidence in 
the Mexican fold-Thrust Belt. Journal of South American Earth Sciences 76: 101-114.

Ramos-Vázquez, M., J.S. Armstrong-Altrin, L. Rosales-Hoz, M.L. MachainCastillo, and A. Carranza-Edwards. 2017. Geochemistry of deep-sea sediments in two cores retrieved at the mouth of the Coatzacoalcos river delta, Western Gulf of Mexico, Mexico. Arabian Journal of Geosciences 10 (6): 148.

Ramos-Vázquez, M.A., and J.S. Armstrong-Altrin. 2019. Sediment chemistry and detrital zircon record in the Bosque and Paseo del mar coastal areas from the southwestern Gulf of Mexico. Marine and Petroleum Geology 110: 650-675.

Ramos-Vázquez, M.A., J.S. Armstrong-Altrin, M.L. Machain-Castillo, and F.R. Gío-Argáez. 2018. Foraminiferal assemblages, ${ }^{14} \mathrm{C}$ ages, and compositional variations in two sediment cores in the western Gulf of Mexico. Journal of South American Earth Sciences 88: 480-496.

Raza, A., and M.E.A. Mondal. 2018. Geochemistry of the Archaean metasedimentary rocks of the Bundelkhand Mauranipur-Babina greenstone belt, Central India: Implications for provenance characteristics. Journal of the Indian Association of Sedimentologists 35 (1): 57-76.

Rivera-Gómez, M.A., J.S. Armstrong-Altrin, S.P. Verma, and L. Díaz-González. 2020. APMDisc: An online computer program for the geochemical discrimination of siliciclastic sediments from active and passive margins. Turkish Journal of Earth Sciences 29: 550-578.

Robin, C. 1982. Mexico. In Andesites, ed. R.S. Thorpe, 137-147. Chichester: Wiley.

Robin, C., and J. Tournon. 1978. Spatial relations of andesitic and alkaline provinces in Mexico and Central America. Canadian Journal of Earth Sciences 15: 1633-1641.

Rosales-Hoz, L., J.J. Kasper-Zubillaga, A. Carranza-Edwards, and O.C. Hernández. 2008. Geochemical composition of surface sediments near Isla de Sacrificios coral reef ecosystem, Veracruz, Mexico. Hidrobiológica 18 (2): 155-165.

Roser, B.P., and R.J. Korsch. 1986. Determination of tectonic setting of sandstone-mudstone suites using $\mathrm{SiO}_{2}$ content and $\mathrm{K}_{2} \mathrm{O} / \mathrm{Na}_{2} \mathrm{O}$ ratio. Journal of Geology 94: 635-650.

Rubatto, D. 2002. Zircon trace element geochemistry: Partitioning with garnet and the link between $\mathrm{U}-\mathrm{Pb}$ ages and metamorphism. Chemical Geology 184: 123-138.

Saha, S., S. Banerjee, S.D. Burley, A. Ghosh, and P.K. Saraswati. 2010. The influence of flood basaltic source terrains on the efficiency of tectonic setting discrimination diagrams: An example from the Gulf of Khambhat, western India. Sedimentary Geology 228: 1-13.

Saha, S., S. Burley, and S. Banerjee. 2018. Mixing processes in modern estuarine sediments from the Gulf of Khambhat, western India. Marine and Petroleum Geology 91: 599-621.

Self, R.P. 1975. Petrologic changes in fluvial sediments in the Rio Nautla drainage basin, Veracruz, Mexico. Journal of Sedimentary Petrology 45 (1): 140-149.

Solari, L.A., A. Gómez-Tuena, F. Ortega-Gutiérrez, and C. Ortega-Obregón. 2011. The Chuacús metamorphic complex, Central Guatemala: Geochronological and geochemical constraints on it PalaeozoicMesozoic evolution. Geologica Acta 9 (3-4): 329-350.

Solari, L.A., C.M. González-León, C. Ortega-Obregón, M. Valencia-Moreno, and M.A. Rascón-Heimpel. 2018. The Proterozoic of NW Mexico revisited: U-Pb geochronology and $\mathrm{Hf}$ isotopes of Sonoran rocks and their tectonic implications. International Journal of Earth Sciences 107: 845-861.

Talavera-Mendoza, O., J. Ruiz, P. Corona-Chavez, G.E. Gehrels, A. SarmientoVillagrana, J.L. García-Díaz, and S.A. Salgado-Souto. 2013. Origin and provenance of basement metasedimentary rocks from the Xolapa complex: New constraints on the Chortis-southern Mexico connection. Earth and Planetary Science Letters 369-370: 188-199.

Tamayo, J.L. 1991. Geografía Moderna de México. 11th ed. México City: Trillas.

Tapia-Fernandez, H.J., J.S. Armstrong-Altrin, and K. Selvaraj. 2017. Geochemistry and U-Pb geochronology of detrital zircons in the Brujas beach sands, Campeche, southwestern Gulf of Mexico, Mexico. Journal of South American Earth Sciences 76: 346-361.
Tawfik, H.A., I.M. Ghandour, W. Maejima, J.S. Armstrong-Altrin, and A.-M.T. Abdel-Hameed. 2017. Petrography and geochemistry of the siliciclastic Araba formation (Cambrian), East Sinai, Egypt: Implications for provenance, tectonic setting and source weathering. Geological Magazine 154 (1): 1-23.

Tawfik, H.A., M.K. Salah, W. Maejima, J.S. Armstrong-Altrin, A.-M.T. AbdelHameed, and M.M.E. Ghandour. 2018. Petrography and geochemistry of the Lower Miocene Moghra sandstones, Qattara depression, North Western Desert, Egypt. Geological Journal 53: 1938-1953.

Taylor, S.R., and S.M. McLennan. 1985. The continental crust: Its composition and evolution. Oxford: Blackwell.

Torres-Sánchez, D., S.K. Verma, S.P. Verma, F. Velasco-Tapia, and J.R. TorresHernández. 2019. Petrogenetic and tectonic implications of OligoceneMiocene volcanic rocks from the sierra de San Miguelito complex, Central Mexico. Journal of South American Earth Sciences 95: 102311.

Udayanapillai, A.V., V. Perumal, and J.S. Armstrong-Altrin. 2020. Provenance, weathering, tectonic setting and palaeo-oxygenation condition of the cretaceous calcareous grey shale (CGS) from the Kallakudi Dalmia limestone quarry no: II, Uttatur group, Trichinopoly, Tamilnadu, India. Himalayan Geology 41 (1): 11-20.

Verma, S.K., E.E.M. Torres, V.P. Malviya, J.R. Torres-Hernández, D. TorresSánchez, B.A. Rivera-Escoto, and P. Mehta. 2019. Geochemistry of Mesozoic volcanic rocks from the Fresnillo area (Chilitos formation), Zacatecas, Mexico: Implications for the magma source and tectonic setting. Journal of South American Earth Sciences 96: 102351.

Verma, S.P. 2005. Estadística Básica para el Manejo de Datos Experimentales: Aplicación a la Geoquímica (Geoquimiometría). Mexico City: National Autonomous University of México (in Spanish).

Verma, S.P. 2009. Continental rift setting for the central part of the Mexican Volcanic Belt: A statistical approach. The Open Geology Journal 3: 8-29.

Verma, S.P. 2015. Origin, evolution, and tectonic setting of the eastern part of the Mexican Volcanic Belt and comparison with the central American volcanic arc from conventional multielement normalized and new multidimensional discrimination diagrams and discordancy and significance tests. Turkish Journal of Earth Sciences 24: 111-164.

Verma, S.P., and J.S. Armstrong-Altrin. 2013. New multi-dimensional diagrams for tectonic discrimination of siliciclastic sediments and their application to Precambrian basins. Chemical Geology 355: 117-180.

Verma, S.P., and J.S. Armstrong-Altrin. 2016. Geochemical discrimination of siliciclastic sediments from active and passive margin settings. Sedimentary Geology 332: 1-12.

Verma, S.P., K. Pandarinath, and M.A. Rivera-Gómez. 2016. Evaluation of ongoing rifting and subduction processes in the geochemistry of magmas from the western part of the Mexican Volcanic Belt. Journal of South American Earth Sciences 66: 125-148.

Vos, K., N. Vandenberghe, and J. Elsen. 2014. Surface textural analysis of quartz grains by scanning electron microscopy (SEM): From sample preparation to environmental interpretation. Earth Science Reviews 128: 93-104.

Wang, J., C. Wu, Z. Li, W. Zhu, T. Zhou, J. Wu, and J. Wang. 2017. Whole-rock geochemistry and zircon $\mathrm{Hf}$ isotope of late carboniferous-Triassic sediments in the Bogda region, NW China: Clues for provenance and tectonic setting. Geological Journal 54: 1853-1877.

Wang, Z., J. Wang, X. Fu, X. Feng, D. Wang, C. Song, W. Chen, S. Zeng, and F. Yu. 2018a. Provenance and tectonic setting of the Quemoco sandstones in the north Qiangtang Basin, North Tibet: Evidence from geochemistry and detrital zircon geochronology. Geological Journal 53 : 1465-1481.

Wang, Z., J. Wang, X. Fu, W. Zhan, J.S. Armstrong-Altrin, F. Yu, X. Feng, C. Song, and S. Zeng. 2018b. Geochemistry of the upper Triassic black mudstones in the Qiangtang Basin, Tibet: Implications for paleoenvironment, provenance, and tectonic setting. Journal of Asian Earth Sciences 160: 118-135.

Weber, B., R. González-Guzmán, R. Manjarrez-Juárez, A. Cisneros de Leon, U. Mertens, L. Solari, L. Hecht, and V. Valencia. 2018. Late Mesoproterozoic to early Paleozoic history of metamorphic basement from the southeastern Chiapas massif complex, Mexico, and implications for the evolution of NW Gondwana. Lithos 300-301: 177-199. 
Weber, B., E.E. Scherer, U.K. Martens, and K. Mezger. 2012. Where did the lower Paleozoic rocks of Yucatan come from? A U-Pb, Lu-Hf, and Sm$\mathrm{Nd}$ isotope study. Chemical Geology 312-313: 1-17.

Weber, B., V.A. Valencia, P. Schaaf, and F. Ortega-Gutiérrez. 2009. Detrital zircon ages from the lower Santa Rosa formation, Chiapas: Implications on regional Paleozoic stratigraphy. Revista Mexicana de Ciencias Geologicas 26: 260-276.

Wengler, M., J.R. Barboza-Gudiño, T.B. Thomsen, and G. Meinhold. 2019. Sediment provenance of Triassic and Jurassic sandstones in Central Mexico during activity of the Nazas volcanic arc. Journal of South American Earth Sciences 92: 329-349.

$\mathrm{Xu}, \mathrm{H}$. ., B. Liu, and F. Wu. 2010. Spatial and temporal variations of Rb/Sr ratios of the bulk surface sediments in Lake Qinghai. Geochemistry Transactions 11: 3. https://doi.org/10.1186/1467-4866-11-3.

Yañez-Arancibia, A., and J.W. Day Jr. 1982. Ecological characterization of Terminos lagoon: A tropical lagoon estuarine system in the southern Gulf of Mexico. Oceanologica Acta 5: 431-500.

Zhang, Z., Y. Zhou, T. Zhou, S. Yu, Y. He, and F. Gao. 2018. Geochemistry of siltstones of the early cretaceous Laiyang Group in Taolin area, Shandong Province, eastern China: Implications for provenance, source weathering, palaeo-environment, and tectonic setting. Geological Journal 53: 1-14.

\section{Publisher's Note}

Springer Nature remains neutral with regard to jurisdictional claims in published maps and institutional affiliations.

\section{Submit your manuscript to a SpringerOpen ${ }^{\circ}$ journal and benefit from:}

- Convenient online submission

- Rigorous peer review

- Open access: articles freely available online

High visibility within the field

- Retaining the copyright to your article

Submit your next manuscript at $\boldsymbol{\nabla}$ springeropen.com 